\title{
Enhancing Faradaic Charge Storage Contribution in Hybrid Pseudocapacitors
}

\author{
Henri-Louis Girard, Hainan Wang, Anna d'Entremont and Laurent Pilon* \\ University of California, Los Angeles, \\ Henry Samueli School of Engineering and Applied Science, \\ Mechanical and Aerospace Engineering Department, \\ 420 Westwood Plaza, \\ Los Angeles, CA 90095, USA
}

September 6, 2015

\footnotetext{
${ }^{*}$ Corresponding author, Email: pilon@seas.ucla.edu, Tel: +1 (310) 206-5598, Fax: +1 (310) 2062302

Preprint submitted to Elsevier

September 6, 2015

(C) 2015. This manuscript version is made available under the Elsevier user license http://www.elsevier.com/open-access/userlicense/1.0/
} 


\section{Nomenclature}

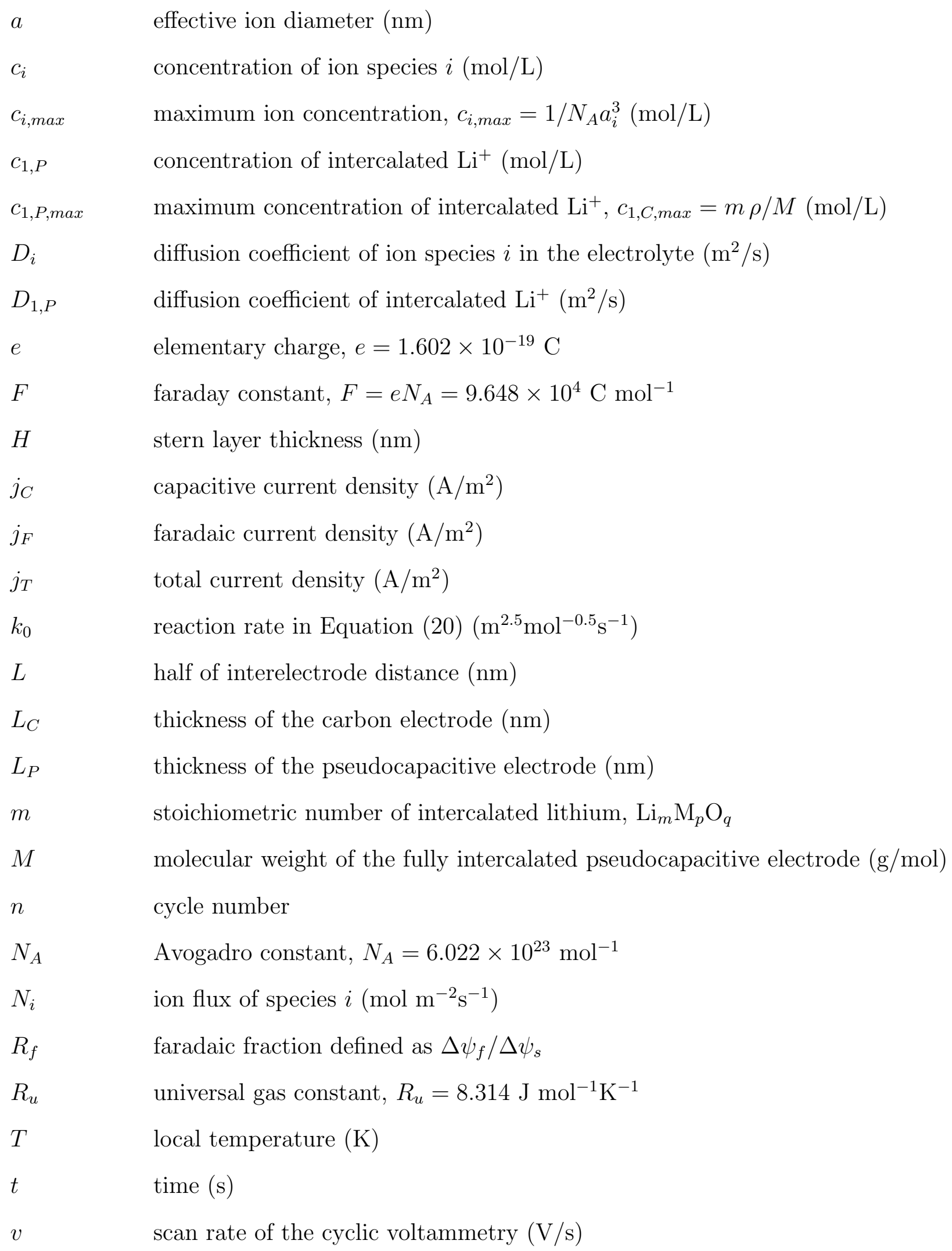


$x \quad$ location in one-dimensional space $(\mu \mathrm{m})$

$z_{i} \quad$ ion valency

\section{Greek symbols}

$\alpha \quad$ transfer coefficient in Equation (20)

$\epsilon_{0} \quad$ vacuum permittivity, $\epsilon_{0}=8.854 \times 10^{-12} \mathrm{~F} \mathrm{~m}^{-1}$

$\epsilon_{r} \quad$ relative permittivity of electrolyte

$\Delta \psi_{e q} \quad$ equilibrium potential difference (V)

$\Delta \psi_{f} \quad$ faradaic potential window defined as $\psi_{f}-\psi_{\min }(\mathrm{V})$

$\Delta \psi_{H} \quad$ potential drop across the Stern layer (V)

$\Delta \psi_{s} \quad$ potential window $(\mathrm{V})$

$\lambda_{D} \quad$ Debye length $(\mathrm{m})$

$\eta \quad$ overpotential, $\eta=\Delta \psi_{H}-\Delta \psi_{e q}(\mathrm{~V})$

$\rho \quad$ density of the fully intercalated pseudocapacitive electrode $\left(\mathrm{kg} / \mathrm{m}^{3}\right)$

$\sigma_{C} \quad$ electrical conductivity of the carbon electrode $(\mathrm{S} / \mathrm{m})$

$\sigma_{P} \quad$ electrical conductivity of the pseudocapacitive electrode $(\mathrm{S} / \mathrm{m})$

$\tau_{C V} \quad$ cycle period (s)

$\tau_{d} \quad$ diffusion time scale across the EDL, $\tau_{d}=\lambda_{d}^{2} / D_{1}(\mathrm{~s})$

$\tau_{t h} \quad$ thermal potential, $\tau_{t h}=R_{u} T / z_{1} F(\mathrm{~s})$

$\psi \quad$ local electric potential (V)

$\psi_{f} \quad$ potential associated with the $\mathrm{Li}^{+}$starvation (V)

$\psi_{s} \quad$ imposed cell potential (V)

\section{Superscripts and subscripts}

* $\quad$ refers to dimensionless variable

$\infty \quad$ refers to bulk electrolyte

$i \quad$ refers to ion species $i$

C refers to the carbon electrode

$P \quad$ refers to the pseudocapacitive electrode 


\section{Introduction}

Electrochemical capacitors are promising devices for electrical energy storage in particular for high power application because of their very large power density compared with batteries [1-3]. They are usually classified as either electric double layer capacitors (EDLCs) or pseudocapacitors. EDLCs store charge physically via the formation of an electric double layer (EDL) at the interface between the electrode and the electrolyte [1-3]. This phenomenon involves the fast diffusion of ions in the electrolyte responsible for their high power density. However, EDLCs have relatively low energy density compared with batteries. By contrast, pseudocapacitors can store charge not only via the formation of an EDL but also via reversible oxidation-reduction (redox) reactions with fast insertion of the reaction products in the electrode $[1,3-6]$. Because the electrical energy is stored chemically in the volume of the electrode, pseudocapacitors tend to feature larger energy density than EDLCs. They also maintain high power density as the behavior of the cell is typically capacitive even though the charge storage mechanism is faradaic [6]. Finally, hybrid pseudocapacitors combine a pseudocapacitive electrode, typically made of transition metal oxide, providing high energy density, and an EDLC-type electrode, made of carbon, enabling large power density [2, 7-11]. For this reason, hybrid pseudocapacitors have been extensively studied both experimentally $[2,6-11]$ and numerically [12-21].

Optimizing the pseudocapacitive electrode in hybrid pseudocapacitors is made difficult by the interdependent physical phenomena involved. Indeed, thicker electrodes offer more volume to store charge but the typically low electrical conductivity of transition metal oxides is responsible for a potential drop across the electrode that limits the thickness that can be used in practice. In addition, the relatively small solid-state diffusion coefficient associated with intercalation inside the electrode also limits the performance of the electrode, in particular for fast cycling or high power applications. Moreover, it is very challenging to discriminate experimentally the contribution to charge storage of the faradaic reactions from that of the EDL formation. However, this can be achieved through numerical simulations [22]. In fact, it has been established numerically that hybrid pseudocapacitors operate under 
two distinct regimes: a faradaic and a capacitive regime. The first is dominated by charge storage via faradaic reactions while the second is dominated by EDL formation albeit with smaller current density.

Numerical simulations can also be used to identify the conditions that maximize the faradaic contribution leading to larger total current densities and areal capacitance. Several models for pseudocapacitors have been developed based on equivalent RC circuit models [2325], molecular dynamics simulations [26-30], and continuum modeling [12-21]. In particular, the Generalized Modified Poisson-Nernst-Planck (GMPNP) model accounts simultaneously for EDL formation by electromigration of an arbitrary number of ions with different finite sizes, valencies and diffusion coefficients. It has been used along with models for redox reactions and insertion of the reaction product to predict trends and to provide physical interpretation in hybrid pseudocapacitors [22]. In particular, $\mathrm{Li}^{+}$starvation at the electrode/electrolyte interface was shown to be responsible for the transition from the faradaic to the capacitive regime. This transition also corresponded to the drop in $b$-value often observed experimentally [31-33]. Indeed, up to that point, the faradaic reaction consumed $\mathrm{Li}^{+}$ accumulated at the surface and was therefore diffusion-independent. In this case, the device had a capacitive behavior and the b-value was unity. After $\mathrm{Li}^{+}$starvation, however, $\mathrm{Li}^{+}$ions had to diffuse from the inner electrode to reach the reaction front at the electrode/electrolyte interface. Then, the faradaic reaction was diffusion-controlled and the b-value was less than one $[22]$.

Scaling analysis is a powerful tool used to simplify complex models and to reduce the number of variables by non-dimensionalizing the governing equations and the initial and boundary conditions. This process typically leads to the introduction of dimensionless numbers or similarity parameters comparing competing physical phenomena. It can also be used to identify different regimes of operation and to uncover scaling laws or correlations governing the evolution of various parameters of interest. These correlations can then be used to design or to operate a device in the most favorable regime. For example, Wang and co-workers $[34,35]$ identified a criteria for the transition between diffusion-limited and 
diffusion-independent regimes in cyclic voltammetry for EDLCs with binary symmetric [34] and asymmetric electrolyte [35]. They also performed scaling analysis to identify a selfsimilar behavior for the EDL capacitance for EDLCs with binary and symmetric electrolytes under equilibrium conditions [34]. They then derived a correlation relating the electrode capacitance to the average pore size and electrolyte properties from experimental data for a wide range of porous carbon electrode morphologies [34].

The objective of this study is to derive design rules for pseudocapacitive electrodes in order to ensure that the hybrid pseudocapacitor operates exclusively in the faradaic regime. To do so, dimensional analysis of the continuum model recently developed - to simulate hybrid pseudocapacitors subjected to cyclic voltammetry [22] - was performed to capture the combined effects of electrode thickness, diffusion coefficient for intercalation, and scan rate.

\section{Analysis}

\subsection{Schematics and Assumptions}

Figure 1 shows the schematic of the one-dimensional (1D) hybrid pseudocapacitor simulated as well as the computational domain and the associated coordinate system. The origin was located at the midplane of the cell. The device consisted of two planar electrodes separated by an electrolyte. The pseudocapacitive electrode had a thickness $L_{P}$ and was made of transition metal oxide $\mathrm{M}_{p} \mathrm{O}_{q}$. The carbon electrode thickness was $L_{C}$. The electrolyte, of thickness $2 \mathrm{~L}$, consisted of $\mathrm{LiClO}_{4}$ salt in propylene carbonate (PC) solvent. It was further divided in three domains: a Stern layer of thickness $H$ near each electrode and a diffuse layer. There was no free charge in the Stern layer and its thickness corresponded to the distance of closest approach of the ions to the electrode. In the diffuse layer, ions were mobile under the effects of electrodiffusion and steric repulsion. At the interface between the pseudocapacitive electrode and the electrolyte, the following reaction was assumed to take place $[6,32]$

$$
m \mathrm{Li}^{+}+\mathrm{M}_{p} \mathrm{O}_{q}+m \mathrm{e}^{-} \rightleftharpoons \mathrm{Li}_{m} \mathrm{M}_{p} \mathrm{O}_{q}
$$

To make the problem mathematically tractable, the following assumptions were made: 
(1) the electrodiffusion of all species was treated as one-dimensional. (2) The Stern layer thickness was approximated as the radius of the largest solvated ion [35-37]. In practice, the ions may lose part of their solvation shell near the electrode. However, to the best of our knowledge, no quantitative model exists to account for this phenomenon. (3) The diffusion coefficients of all ion species in the electrolyte were independent of their respective concentrations. Their values corresponded to those of dilute solutions. Note that in reality the diffusion coefficient of ions varies with concentration. However, when reported, this variation did not exceed $40 \%$ and the effect on the current response was found to be negligible $[22,38]$. (4) The temperature was constant and uniform throughout the device. (5) Advection of the electrolyte was negligible. (6) The redox reaction was heterogeneous and occurred at the pseudocapacitive electrode/electrolyte interface [37]. (7) $\mathrm{Li}^{+}$ion insertion (or intercalation) in the pseudocapacitive electrode was treated as a diffusion process. (8) The redox material did not undergo any phase transition. This assumption is consistent with experimental observations even for thick $\mathrm{Nb}_{2} \mathrm{O}_{5}$ electrodes [39]. (9) Ion adsorption due to non-electrostatic forces was assumed to be negligible. (10) The current collectors were assumed to be perfect conductors such that the potential drop across them was negligible. (11) The relative permittivity of the electrolyte $\epsilon_{r}$ was taken as constant and independent of the electric field. This simplification was necessary to be able to perform dimensional analysis of the governing equations.

\subsection{Generalized Modified Poisson-Nernst-Planck (GMPNP) Model with Faradaic Reactions}

The model used in this study was described in Equations (4) to (22) of Ref. [22] and need not be repeated. It accounts simultaneously for (i) charge transport in the electrodes and electrolyte, (ii) formation and dissolution of the electric double layer (EDL) at the electrode/electrolyte interface, (iii) steric repulsions due to finite ion size, (iv) redox reactions at the pseudocapacitive electrode/electrolyte interface, and (v) insertion and deinsertion of the reaction product in the pseudocapacitive electrode. Mathematically, this model consists of the 1D Poisson equations for the local electrical potential $\psi(x, t)$ in both electrodes and in the electrolyte coupled to the time-dependent 1D mass conservation equations for the ion 
concentrations $c_{1}(x, t)$ and $c_{2}(x, t)$ in the electrolyte, and for the concentration of lithium $c_{1, P}(x, t)$ in the pseudocapacitive electrode. Here, ions species $i=1$ refers to cations $\mathrm{Li}^{+}$of valency $z_{1}=1$ while $i=2$ refers to anions $\mathrm{ClO}_{4}^{-}$of valency $z_{2}=-1$ while subscript " 1 ,P" refers to intercalated lithium. The initial conditions impose (a) a uniform potential, $\psi(x, 0)$ $=0 \mathrm{~V}$ throughout the device, (b) uniform bulk concentrations $c_{1}(x, 0)=c_{2}(x, 0)=c_{i, \infty}=$ $1 \mathrm{~mol} / \mathrm{L}$ in the electrolyte, and (c) a non-zero uniform lithium concentration in the pseudocapacitive electrode $c_{1, P}(x, 0)=c_{1, P, 0}$. Finally, in order to simulate cyclic voltammetry experiments, a triangular potential waveform was imposed at the current collector of the pseudocapacitive electrode with a potential window between $\psi_{\min }$ and $\psi_{\max }$ with respect to the carbon electrode treated as grounded. The ion fluxes vanished at the electrode surfaces except for the reacting species at the pseudocapacitive electrode/electrolyte interface. The rate of the faradaic reaction at this interface was governed by the Frumkin-Butler-Volmer equation [37].

\subsection{Dimensional Analysis}

\subsubsection{Dimensionless Variables}

Based on our previous studies [35, 40], the following scaling parameters were used to scale the governing equations for the potential $\psi(x, t)$, the ion concentrations $c_{i}(x, t)$, and the concentration of intercalated lithium in the pseudocapacitive electrode $c_{1, P}(x, t)$ as well as the associated boundary and initial conditions

$$
x^{*}=\frac{x}{\lambda_{D}}, t^{*}=\frac{t}{\lambda_{D}^{2} / D_{1}}, \psi^{*}=\frac{\psi(x, t)}{R_{u} T / z_{1} F}, c_{i}^{*}=\frac{c_{i}(x, t)}{c_{1, \infty}}, \text { and } c_{1, P}^{*}=\frac{c_{1, P}(x, t)}{c_{1, P, \max }}
$$

where $\lambda_{D}$ is the Debye length for binary and asymmetric electrolytes defined as [36]

$$
\lambda_{D}=\sqrt{\epsilon_{0} \epsilon_{r} R_{u} T / F^{2} \sum_{i=1}^{2} z_{i}^{2} c_{i, \infty}}
$$

It represents an estimate of the electric double layer thickness [41]. The time $t$ was scaled by the timescale for ion diffusion across the EDL given by $\tau_{d}=\lambda_{D}^{2} / D_{1}$ where $D_{1}$ is the diffusion coefficient of the cation $\mathrm{Li}^{+}$in the electrolyte. The potential $\psi(x, t)$ was scaled by the thermal potential $\tau_{t h}=R_{u} T / z_{1} F$ representing the electric potential at which the 
electrical potential energy of one mole of $\mathrm{Li}^{+}$ions is equals to its thermal energy $R_{u} T . R_{u}$ $=8.3145 \mathrm{~J} / \mathrm{molK}$ is the universal gas constant and $F=96485 \mathrm{~s} \mathrm{~A} / \mathrm{mol}$ is the Faraday constant. The ion concentrations $c_{i}(x, t)$ were scaled by the bulk concentration $c_{1, \infty}$ of $\mathrm{Li}^{+}$ in the electrolyte. The lithium concentration in the pseudocapacitive electrode $c_{1, P}(x, t)$ was scaled by the maximum lithium concentration $c_{1, P, \max }$ corresponding to the fully intercalated metal oxide electrode.

\subsubsection{Dimensionless Governing Equations}

The Poisson equations governing the electric potential in the pseudocapactive and carbon electrodes assuming constant electrical conductivities, respectively denoted by $\sigma_{P}$ and $\sigma_{C}$, can be expressed in dimensionless form as

$$
\begin{aligned}
& \frac{\partial}{\partial x^{*}}\left(\sigma_{P}^{*} \frac{\partial \psi^{*}}{\partial x^{*}}\right)=0 \text { for } \quad-\left(L^{*}+L_{P}^{*}\right) \leq x^{*}<-L^{*} \\
& \frac{\partial}{\partial x^{*}}\left(\sigma_{C}^{*} \frac{\partial \psi^{*}}{\partial x^{*}}\right)=0 \text { for } \quad L^{*}<x^{*} \leq L^{*}+L_{C}^{*}
\end{aligned}
$$

where the dimensionless electrode conductivities are given by

$$
\sigma_{P / C}^{*}=\left[\sigma_{P / C}\left(\psi_{\max }-\psi_{\min }\right) / L_{P / C}\right] /\left(F z_{1} c_{1, \infty} D_{1} / L\right)
$$

where subscript $P$ and $C$ refers to the pseudocapacitive and to the carbon electrode, respectively. They represent the ratios of the characteristic electric current density in the pseudocapacitive or carbon electrode to the ionic current density in the electrolyte. Here, $L^{*}=L / \lambda_{D}$ represents the dimensionless electrolyte thickness while $L_{P}^{*}=L_{P} / \lambda_{D}$ and $L_{C}^{*}=L_{C} / \lambda_{D}$ are the dimensionless pseudocapacitive and carbon electrode thicknesses, respectively.

Similarly, the mass conservation equation in the pseudocapacitive electrode was nondimensionalized as

$$
\frac{\partial c_{1, P}^{*}}{\partial t^{*}}=\frac{\partial}{\partial x^{*}}\left(D_{1, P}^{*} \frac{\partial c_{1, P}^{*}}{\partial x^{*}}\right) \quad \text { for } \quad-\left(L^{*}+L_{P}^{*}\right) \leq x^{*} \leq-L^{*}
$$

where $D_{1, P}^{*}=D_{1, P} / D_{1}$ is the ratio of lithium atom diffusion coefficient in the pseudocapacitive electrode $D_{1, P}$ to that of $\mathrm{Li}^{+}$ions in the electrolyte $D_{1}$. 
Moreover, the GMPNP model in the diffuse layer of the binary and asymmetric electrolyte, between $-L^{*}+H^{*}$ and $L^{*}-H^{*}$, has already been written in dimensionless form as $[35]$

$$
\left\{\begin{array}{l}
\frac{\partial^{2} \psi^{*}}{\partial x^{* 2}}=-\frac{c_{1}^{*}+z_{2}^{*} c_{2}^{*}}{1-z_{2}^{*}} \\
\frac{\partial c_{i}^{*}}{\partial t^{*}}=-\frac{\partial N_{i}^{*}}{\partial x^{*}} \quad \text { for } i=1 \text { or } 2
\end{array}\right.
$$

Here, $H^{*}=H / \lambda_{D}=a_{2}^{*} / 2$ where $a_{2}^{*}=a_{2} / \lambda_{D}$ is the effective ion diameter $a_{2}$ of the largest ion $\left(\mathrm{ClO}_{4}^{-}\right.$with $\left.i=2\right)$ scaled by the Debye length $\lambda_{D}$. Additionally, $z_{i}^{*}=z_{i} / z_{1}$ is the ratio of the valency of the ion considered to that of the cation $\mathrm{Li}^{+}(i=1)$. The dimensionless mass flux $N_{i}^{*}$ of ion species $i$ was given by [35]

$$
N_{i}^{*}\left(x^{*}, t^{*}\right)=-D_{i}^{*} \frac{\partial c_{i}^{*}}{\partial x^{*}}-D_{i}^{*} z_{i}^{*} c_{i}^{*} \frac{\partial \psi^{*}}{\partial x^{*}}-\frac{D_{i}^{*} c_{i}^{*}}{2-\sum_{j=1}^{2} \nu_{p j} c_{j}^{*}} \frac{\partial}{\partial x^{*}}\left(\sum_{j=1}^{2} \nu_{p j} c_{j}^{*}\right) \text { for } i=1 \text { or } 2
$$

where $D_{i}^{*}=D_{i} / D_{1}$ is the ratio of the diffusion coefficient of the ion species $i$ and that of the cation $\mathrm{Li}^{+}$. Here, the packing parameter for ion species $i$ is also a dimensionless number defined as $\nu_{p i}=2 c_{i, \infty} / c_{i, \max }$. It represents the ratio of the total bulk ion concentration to the maximum ion concentration $c_{i, \max }=1 / a_{i}^{3} N_{A}$ assuming a simple cubic packing of ions of diameter $a_{i}[42]$.

\subsubsection{Dimensionless Initial and Boundary Conditions}

The initial dimensionless electric potential was uniformly zero across the device, i.e.,

$$
\psi^{*}\left(x^{*}, 0\right)=0 \quad \text { for }-L^{*}-L_{P}^{*} \leq x^{*} \leq L^{*}+L_{C}^{*}
$$

The initial dimensionless concentration of lithium was uniform in the pseudocapacitive electrode such that

$$
c_{1, P}^{*}\left(x^{*}, 0\right)=c_{1, P, 0}^{*}=c_{1, P, 0} / c_{1, P, \max } \quad \text { for } \quad-L^{*}-L_{P}^{*} \leq x^{*} \leq-L^{*}
$$

where $c_{1, P, 0}$ is the concentration of $\mathrm{Li}^{+}$initially intercalated in the pseudocapacitive electrode. 
The concentrations of $\mathrm{Li}^{+}$and $\mathrm{ClO}_{4}^{-}$in the electrolyte were also initially uniform and satisfied electroneutrality so that [35]

$$
c_{1}^{*}\left(x^{*}, 0\right)=1 \text {, and } c_{2}^{*}\left(x^{*}, 0\right)=-1 / z_{2}^{*} \quad \text { for } \quad-L^{*}+a_{2}^{*} / 2 \leq x^{*} \leq L^{*}-a_{2}^{*} / 2 .
$$

The dimensionless electric potentials imposed at the current collector/electrode interfaces were given by [35],

$$
\psi^{*}\left(L^{*}+L_{C}^{*}, t^{*}\right)=0 \quad \text { and } \quad \psi^{*}\left(-L^{*}-L_{P}^{*}, t^{*}\right)=\psi_{s}^{*}\left(t^{*}\right)
$$

where $\psi_{s}^{*}\left(t^{*}\right)$ is the dimensionless surface potential. For cyclic voltammetry, it is given by [35]

$\psi_{s}^{*}\left(t^{*}\right)= \begin{cases}\psi_{\max }^{*}-v^{*}\left[t^{*}-\left(n_{C}-1\right) \tau_{C V}^{*}\right] & \text { for }\left(n_{C}-1\right) \tau_{C V}^{*} \leq t^{*} \leq\left(n_{C}-1 / 2\right) \tau_{C V}^{*} \\ \psi_{\min }^{*}+v^{*}\left[t^{*}-\left(n_{C}-1 / 2\right) \tau_{C V}^{*}\right] & \text { for }\left(n_{C}-1 / 2\right) \tau_{C V}^{*} \leq t^{*} \leq n_{C} \tau_{C V}^{*}\end{cases}$

where $n_{C}$ is the cycle number and $\psi_{\max }^{*}=\psi_{\max } /\left(R_{u} T / z_{1} F\right)$ and $\psi_{\min }^{*}=\psi_{\min } /\left(R_{u} T / z_{1} F\right)$ are the maximum and minimum dimensionless surface potentials, respectively. Here, the dimensionless scan rate $v^{*}=\left(\lambda_{D}^{2} / D_{1}\right) /\left[\left(R_{u} T / z_{1} F\right) / v\right]$ can be interpreted as the ratio of the timescale for ion diffusion across the EDL $\tau_{d}=\lambda_{D}^{2} / D_{1}$ and the characteristic time $\tau_{\text {th }}=\left(R_{u} T / z_{1} F\right) / v$ necessary to reach the thermal potential at scan rate $v$, i.e., $v^{*}=\tau_{d} / \tau_{\text {th }}$ [35]. Finally, $\tau_{C V}^{*}=\tau_{C V} / \tau_{d}=\left(\psi_{\max }^{*}-\psi_{\min }^{*}\right) / v^{*}$ represents the dimensionless cycle period.

Moreover, the boundary conditions accounting for the Stern layer at both electrodes can be written in dimensionless form as [35],

$$
\begin{array}{rrr}
\frac{\partial \psi^{*}}{\partial x^{*}}\left(L^{*}-a_{2}^{*} / 2, t^{*}\right) & =\quad \frac{2}{a_{2}^{*}}\left[\psi^{*}\left(L^{*}, t^{*}\right)-\psi^{*}\left(L^{*}-a_{2}^{*} / 2, t^{*}\right)\right] \\
-\frac{\partial \psi^{*}}{\partial x^{*}}\left(-L^{*}+a_{2}^{*} / 2, t^{*}\right) & =\frac{2}{a_{2}^{*}}\left[\psi^{*}\left(-L^{*}, t^{*}\right)-\psi^{*}\left(-L^{*}+a_{2}^{*} / 2, t^{*}\right)\right] .
\end{array}
$$

The boundary conditions ensuring the continuity of the current density across the pseudocapacitive electrode/electrolyte interface can be written in dimensionless form as

$$
-\frac{\sigma_{P}^{*}}{\psi_{\text {max }}^{*}-\psi_{\min }^{*}} \frac{L_{P}^{*}}{L^{*}} \frac{\partial \psi^{*}}{\partial x^{*}}\left(-L^{*}, t^{*}\right)=j_{C}^{*}\left(-L^{*}+a_{2}^{*} / 2, t^{*}\right)+j_{F}^{*}\left(t^{*}\right)
$$

Similarly, across the carbon electrode/electrolyte interface [35]

$$
-\frac{\sigma_{C}^{*}}{\psi_{\max }^{*}-\psi_{\min }^{*}} \frac{L_{C}^{*}}{L^{*}} \frac{\partial \psi^{*}}{\partial x^{*}}\left(L^{*}, t^{*}\right)=j_{C}^{*}\left(L^{*}-a_{2}^{*} / 2, t^{*}\right) .
$$




$$
\begin{aligned}
j_{C}( \pm(L-H), t) & =-\epsilon_{0} \epsilon_{r} \frac{\partial^{2} \psi}{\partial x \partial t}( \pm(L-H), t) \\
j_{F}(t) & =j_{F, 0}(t)\left[\exp \left(\frac{(1-\alpha) z_{1} F \eta}{R_{u} T}\right)-\exp \left(\frac{-\alpha z_{1} F \eta}{R_{u} T}\right)\right]
\end{aligned}
$$

where $\alpha$ is the transfer coefficient. The surface overpotential $\eta(t)$ is defined as $\eta(t)=$ $\Delta \psi_{H}(t)-\Delta \psi_{e q}$ where $\Delta \psi_{H}(t)=\psi(-L, t)-\psi(-L+H, t)$ is the electrical potential drop across the Stern layer near the pseudocapacitive electrode and $\Delta \psi_{e q}$ is the equilibrium potential difference associated with the redox reaction [37]. Finally, the exchange current density $j_{F, 0}(t)$ is expressed as [22]

$$
j_{F, 0}(t)=F z_{1} k_{0}\left[c_{1}(-L+H, t)\right]^{1-\alpha}\left[c_{1, P, \max }-c_{1, P}(-L, t)\right]^{\alpha}\left[c_{1, P}(-L, t)\right]^{\alpha}
$$

where $k_{0}$ is the reaction rate constant expressed in $\mathrm{m}^{1+3 \alpha} \mathrm{mol}^{-\alpha} \mathrm{S}^{-1}$.

Both current densities $j_{C}(x, t)$ and $j_{F}(t)$ can be scaled by a characteristic ionic current density $F z_{1} D_{1} c_{1, \infty} / \lambda_{D}$ corresponding to diffusion of $\mathrm{Li}^{+}(i=1)$ over the characteristic concentration gradient $c_{1, \infty} / \lambda_{D}$ to yield the dimensionless current densities

$$
j_{C}^{*}\left(x^{*}, t^{*}\right)=\frac{j_{C}(t)}{F z_{1} D_{1} c_{1, \infty} / \lambda_{D}} \quad \text { and } \quad j_{F}^{*}\left(t^{*}\right)=\frac{j_{F}(t)}{F z_{1} D_{1} c_{1, \infty} / \lambda_{D}} .
$$

These dimensionless current densities can be expressed as

$$
\begin{aligned}
j_{C}^{*}\left( \pm\left(L^{*}-a_{2}^{*} / 2\right), t^{*}\right) & =-\left(1-z_{2}^{*}\right) \frac{\partial^{2} \psi^{*}}{\partial x^{*} \partial t^{*}}\left( \pm\left(L^{*}-a_{2}^{*} / 2\right), t^{*}\right) \\
\text { and } j_{F}^{*}\left(t^{*}\right) & =j_{F, 0}^{*}\left(t^{*}\right)\left\{\exp \left[(1-\alpha) \eta^{*}\right]-\exp \left[-\alpha \eta^{*}\right]\right\} .
\end{aligned}
$$

Here, $\eta^{*}$ is the dimensionless surface overpotential defined as $\eta^{*}=\Delta \psi_{H}^{*}\left(t^{*}\right)-\Delta \psi_{e q}^{*}$ and the dimensionless exchange current density $j_{F, 0}^{*}$ can be expressed as

$$
j_{F, 0}^{*}\left(t^{*}\right)=k_{0}^{*} c_{1, P, \max }^{*} c_{1}^{*}\left(-L^{*}+H^{*}, t^{*}\right)^{1-\alpha}\left(1-c_{1, P}^{*}\left(-L^{*}, t^{*}\right)\right)^{\alpha}\left(c_{1, P}^{*}\left(-L^{*}, t^{*}\right)\right)^{\alpha}
$$

where the dimensionless reaction rate constant is expressed as $k_{0}^{*}=k_{0} c_{1, \infty}^{\alpha} \lambda_{D} / D_{1}$ and can be interpreted as the ratio of the timescale for ion diffusion across the EDL $\tau_{d}=\lambda_{D}^{2} / D_{1}$ to the redox reaction timescale given by $\tau_{f}=\lambda_{D} / k_{0} c_{1, \infty}^{\alpha}$. 
In addition, the dimensionless mass fluxes of $\mathrm{Li}^{+}(i=1)$ and $\mathrm{ClO}_{4}^{-}(i=2)$ at the Stern/diffuse layer interface near the pseudocapacitive electrode satisfied

$$
N_{1}^{*}\left(-L^{*}+a_{2}^{*} / 2, t^{*}\right)=j_{F}^{*}\left(t^{*}\right) \quad \text { and } \quad N_{2}^{*}\left(-L^{*}+a_{2}^{*} / 2, t^{*}\right)=0 .
$$

However, at the carbon electrode Stern/diffuse layer interface both ion fluxes vanish so that

$$
N_{1}^{*}\left(L^{*}-a_{2}^{*} / 2, t^{*}\right)=N_{2}^{*}\left(L^{*}-a_{2}^{*} / 2, t^{*}\right)=0 .
$$

Overall, the scaling analysis indicates that the dimensionless governing equations and associated boundary and initial conditions are governed by seventeen dimensionless similarity parameters expressed as

$$
\begin{aligned}
& v^{*}=\frac{\lambda_{D}^{2} / D_{1}}{\left(R_{u} T / z_{1} F\right) / v}, \quad \psi_{\min }^{*}=\frac{\psi_{\min }}{R_{u} T / z_{1} F}, \quad \psi_{\max }^{*}=\frac{\psi_{\max }}{R_{u} T / z_{1} F}, \quad L^{*}=\frac{L}{\lambda_{D}}, \\
& a_{2}^{*}=\frac{a_{2}}{\lambda_{D}}, \quad \nu_{p 1}=2 a_{1}^{3} N_{A} c_{1, \infty}, \quad \nu_{p 2}=2 a_{2}^{3} N_{A} c_{1, \infty}, \quad D_{2}^{*}=\frac{D_{2}}{D_{1}}, \quad z_{2}^{*}=\frac{z_{2}}{z_{1}}, \\
& \sigma_{P}^{*}=\frac{\sigma_{P}\left(\psi_{\max }-\psi_{\min }\right) / L_{P}}{F z_{1} c_{1, \infty} D_{1} / L}, \quad \sigma_{C}^{*}=\frac{\sigma_{C}\left(\psi_{\max }-\psi_{\min }\right) / L_{C}}{F z_{1} c_{1, \infty} D_{1} / L}, \\
& L_{P}^{*}=\frac{L_{P}}{\lambda_{D}}, \quad L_{C}^{*}=\frac{L_{C}}{\lambda_{D}}, \quad D_{1, P}^{*}=\frac{D_{1, P}}{D_{1}}, \quad c_{1, P, \max }^{*}=\frac{c_{1, P, \max }}{c_{1, \infty}}, \\
& k_{0}^{*}=\frac{k_{0} c_{1, \infty}^{\alpha} \lambda_{D}}{D_{1}}, \quad \text { and } \quad \Delta \psi_{e q}^{*}=\frac{\Delta \psi_{e q}}{R_{u} T / z_{1} F} .
\end{aligned}
$$

Note that $v^{*}, \psi_{\min }^{*}, \psi_{\max }^{*}, L^{*}, a_{2}^{*}, \nu_{p 1}, \nu_{p 2}, D_{2}^{*}, z_{2}^{*}, \sigma_{P}^{*}, \sigma_{C}^{*}, L_{P}^{*}$, and $L_{C}^{*}$ were identical to those identified in Ref. [35] for CV simulations of electric double layer capacitors with binary and asymmetric electrolytes. For hybrid supercapacitors with redox reactions and intercalation in the pseudocapacitive electrode, four additional dimensionless numbers appear, namely, $D_{1, P}^{*}, c_{1, P, \max }^{*}, k_{0}^{*}$, and $\Delta \psi_{e q}^{*}$.

\subsubsection{Constitutive Relationships}

A total of 22 input parameters were needed to solve the dimensional governing Equations (4) to (22) of Ref.[22] including (i) the electrolyte properties $\epsilon_{r}, a_{1}, a_{2}, z_{1}, z_{2}, D_{1}, D_{2}$, and $c_{1, \infty}$, (ii) the carbon and pseudocapacitive electrode properties $\sigma_{C}, \sigma_{P}, c_{1, P, 0}$ and $D_{1, P}$, (iii) the redox reaction parameters $\alpha, k_{0}$ and $\Delta \psi_{e q}$, (iv) the cell dimensions $L, L_{P}$, and $L_{C}$, and (v) the operating conditions $T, \psi_{\max }, \psi_{\min }$, and $v$. In the present study, the values and ranges of 
the parameters were taken from the literature whenever available and are reported in Table 1. In particular, the electrode parameters $L_{P}$ and $D_{1, P}$ were taken as variables within a range of reasonable values. Furthermore, to evaluate the rate behavior of the device, the scan rate $v$ was also varied. The transfer coefficient of the redox reaction $\alpha$ is generally assumed to be $1 / 2$ corresponding to equal energy barriers for forward and backward redox reactions [37]. The baseline case corresponded to Case $\mathrm{B}$ of Ref.[22] considering a $\mathrm{Nb}_{2} \mathrm{O}_{5}$ pseudocapacitive electrode and a carbon electrode separated by an electrolyte consisting of $\mathrm{LiClO}_{4}$ salt in organic solvent propylene carbonate (PC) at $1 \mathrm{M}$. Note that we verified that the cell charging/discharging was not limited by ion diffusion in the electrolyte by increasing the diffusion coefficients $D_{1}$ and $D_{2}$ of $\mathrm{Li}^{+}$and $\mathrm{ClO}_{4}^{-}$by a factor of 10 without seeing any change in the predicted potential, concentrations and currents.

\subsubsection{Method of Solution and Data Processing}

The dimensional governing Equations (4) to (22) of Ref.[22] were solved using the commercial finite element solver COMSOL $4.3 \mathrm{~b}$ run in parallel on 8 cores with 64 GB of RAM. The numerical convergence was considered to be reached when the maximum relative difference in the predicted current densities $j_{C}$ and $j_{F}$ at the Stern/diffuse layer interface near the pseudocapacitive electrode $(x=-L+H)$ was less than $1 \%$ when dividing both the mesh size and the time step by a factor two. This led to imposing a time step of $\Delta t \approx \tau_{C V} / 1000=\left(\psi_{\max }-\psi_{\min }\right) / 1000 v$. The mesh size was the smallest at the electrode/electrolyte interface due to the large potential and concentration gradients and was gradually increased further from the electrode. The mesh size was specified to be less than $0.025 \mathrm{~nm}$ at the Stern/diffuse layer interface while it was less than $L / 50$ in the diffuse layer and less than $L_{P} / 25$ and $L_{C} / 25$ in the electrode domains. The total number of finite elements was about 500 for all cases simulated in the present study.

The instantaneous total current density $j_{T}(t)$ at the pseudocapacitive electrode/electrolyte interface was computed as the sum of the capacitive and faradaic current densities, i.e., $j_{T}(t)=j_{C}(t)+j_{F}(t)$. CV curves at a specific scan rate were obtained by plotting $j_{T}(t)$, $j_{C}(t)$, and $j_{F}(t)$ as a function of $\psi_{s}(t)$. Finally, the areal integral capacitance of the device 
$C_{s, i n t}\left(\right.$ in $\left.\mathrm{F} / \mathrm{cm}^{2}\right)$ was computed from the total current $j_{T}(t)$ and the imposed potential $\psi_{s}(t)$ according to [40]

$$
C_{s, \text { int }}=\frac{1}{\psi_{\max }-\psi_{\min }} \oint \frac{j_{T}\left(\psi_{s}\right)}{2 v} \mathrm{~d} \psi_{s} .
$$




\section{Results and Discussion}

\subsection{Faradaic Potential Window}

Figure 2 shows (a) the capacitive $j_{C}(t)$, faradaic $j_{F}(t)$, and total $j_{T}(t)$ current densities as well as (b) the intercalated $\mathrm{Li}^{+}$concentration $c_{1, P}(-L, t)$ at the pseudocapacitive electrode/electrolyte interface as functions of cell potential $\psi_{s}(t)$ between $\psi_{\min }=-0.8 \mathrm{~V}$ and $\psi_{\max }=+0.8 \mathrm{~V}$ at a scan rate $v$ of $1 \mathrm{~V} / \mathrm{s}$. The results were obtained for pseudocapacitive electrode thickness $L_{P}=50 \mathrm{~nm}$ and for $\mathrm{Li}^{+}$diffusion coefficient $D_{1, P}=10^{-14} \mathrm{~m}^{2} / \mathrm{s}$. Figure 2a demonstrates that the cell exhibited two distinct regimes. In the lower part of the potential window, the faradaic regime prevailed as the faradaic current dominated, i.e., $j_{T}(t) \approx j_{F}(t)$. In the higher part of the potential window, the capacitive regime was observed as the capacitive current $j_{C}$ contributed the most to the total current density $j_{T}$. In the discharging step, the transition between the faradaic and capacitive regimes was caused by the slow outward diffusion of $\mathrm{Li}^{+}$in the pseudocapacitive electrode causing $\mathrm{Li}^{+}$starvation at the electrode/electrolyte interface, as illustrated in Figure 2b. In the charging step, the transition from capacitive to faradaic regime, marked by a red square, corresponded to steep increase in the absolute value of the total current density observed on Figure 2a. Note that this transition also corresponded to the end of the dissolution of the $\mathrm{ClO}_{4}^{-}$EDL. Then, the capacitive current vanished during the entire faradaic regime.

The faradaic potential window, represented by a double arrow in Figure 2, was defined as $\Delta \psi_{f}=\psi_{f}-\psi_{\min }$ where $\psi_{f}$ is the potential at which $\mathrm{Li}^{+}$starvation occurs during discharging, as shown by the onset of the capacitive current (blue circle in Figure 2). Experimentally, it may be easier to determine the faradaic potential window by the sharp increase in current density $j_{T}(t)$ in the charging step. Either definition led to very similar faradaic potential window as illustrated by the proximity of the red square and blue circle in Figure 2b. More importantly, the magnitude of the total current was significantly smaller in the capacitive regime than in the faradaic regime. This stresses the need for widening the faradaic potential window by delaying or eliminating $\mathrm{Li}^{+}$starvation. In addition, eliminating $\mathrm{Li}^{+}$starvation would eliminate the transition from the faradaic to the capacitive regime. This would elim- 
inate the dip in $b$-value caused by the transition and improve high rate performance [22].

\subsection{Effect of the Pseudocapacitive Electrode Thickness}

Figure 3 shows (a) the total current density $j_{T}$ and (b) the intercalated $\mathrm{Li}^{+}$concentration $c_{1, P}(-L, t)$ at the pseudocapacitive electrode/electrolyte interface as functions of the cell potential $\psi_{s}(t)$ for pseudocapacitive electrode thickness ranging from $L_{P}=50$ to $500 \mathrm{~nm}$ in the case of a relatively small $\mathrm{Li}^{+}$diffusion coefficient $D_{1, P}=10^{-14} \mathrm{~m}^{2} / \mathrm{s}$. Here also, the scan rate was $1 \mathrm{~V} / \mathrm{s}$. Figure $3 \mathrm{a}$ indicates that thinner electrodes resulted in larger current densities and larger capacitances. In fact, the device with a pseudocapacitive electrode of thickness $L_{P}=50 \mathrm{~nm}$ had an areal integral capacitance $C_{s, i n t}$ of $40 \mu \mathrm{F} / \mathrm{cm}^{2}$. This was almost twice as large as that with electrode thickness $L_{P}=500 \mathrm{~nm}$ found to be $C_{s, \text { int }}=25 \mu \mathrm{F} / \mathrm{cm}^{2}$. Figure $3 \mathrm{a}$ also illustrates that the CV curves exhibited similar peaks for all thicknesses considered. The most prominent peak was observed during charging and indicated the onset of the faradaic charging as indicated in Figure $3 \mathrm{~b}$ by the sharp rise in $c_{1, P}(-L, t)$ as $\mathrm{Li}^{+}$ started intercalating in the electrode. This peak was found to shift toward larger potentials from $\psi_{s}=-0.3 \mathrm{~V}$ to $\psi_{s}=+0.1 \mathrm{~V}$ as the pseudocapacitive electrode thickness decreased. Similarly, the hump in the discharging current also shifted from lower to higher potentials as the electrode thickness decreased. In other words, the faradaic potential window $\Delta \psi_{f}$ widened as the electrode thickness $L_{P}$ decreased.

Figure 3b indicates that thinner electrodes led to larger maximum concentration of intercalated $\mathrm{Li}^{+}$at the electrode surface at the end of the charging step. This was due to (i) the wider fraction of the potential window dominated by faradaic reactions, (ii) the large faradaic current density, and (iii) the smaller electrode volume in which $\mathrm{Li}^{+}$ions could intercalate. Thicker electrodes provided more volume for $\mathrm{Li}^{+}$insertion but suffered from a narrower faradaic potential window and a smaller current magnitude. Figure 3b also features hysteresis in the concentration $c_{1, P}(-L, t)$ suggesting that charging and discharging were limited by slow $\mathrm{Li}^{+}$intercalation caused by the relatively small value of $D_{1, P}$. Note that, in all cases, the $\mathrm{Li}^{+}$concentration $c_{1, P}$ remained significantly smaller than its theoretical maximum of $c_{1, P, \max }=37 \mathrm{~mol} / \mathrm{L}$, corresponding the the fully intercalated electrode. 
Overall, this section has demonstrated that, in the case of hybrid pseudocapacitors limited by $\mathrm{Li}^{+}$intercalation in the pseudocapacitive electrode, a thinner electrode led to larger faradaic potential window. In turn, this led to larger current densities and overall larger areal capacitance by delaying the $\mathrm{Li}^{+}$starvation during the discharging step.

\subsection{Effect of the Intercalated $\mathrm{Li}^{+}$Diffusion Coefficient}

Figure 4 shows (a) the total current density $j_{T}$ as well as (b) the $\mathrm{Li}^{+}$concentration $c_{1, P}(-L, t)$ at the pseudocapacitive electrode/electrolyte interface as functions of cell potential $\psi_{s}(t)$ at a scan rate $v$ of $1 \mathrm{~V} / \mathrm{s}$ for a relatively thick electrode $\left(\mathrm{L}_{P}=500 \mathrm{~nm}\right)$ and for intercalated $\mathrm{Li}^{+}$diffusion coefficients $D_{1, P}$ ranging from $10^{-14}$ to $10^{-11} \mathrm{~m}^{2} / \mathrm{s}$. Figure 4a indicates that the current peak observed during charging increased in magnitude and shifted from $\psi_{s}=-0.25 \mathrm{~V}$ to $\psi_{s}=0 \mathrm{~V}$ as the diffusion coefficient $D_{1, P}$ increased. Similarly, the peak observed during discharging also shifted toward higher potential with increasing diffusion coefficient. This suggests that the faradaic potential window $\Delta \psi_{f}=\psi_{f}-\psi_{\min }$ widened and $\mathrm{Li}^{+}$starvation was delayed as the diffusion coefficient $D_{1, P}$ increased. In fact, the device's areal integral capacitance increased from 25 to $47 \mu \mathrm{F} / \mathrm{cm}^{2}$ as $D_{1, P}$ increased from $10^{-14}$ to $10^{-11} \mathrm{~m}^{2} / \mathrm{s}$. Note that the maximum $\mathrm{Li}^{+}$concentration $c_{1, P}(-L, t)$ reached at the end of the charging step at the electrode/electrolyte interface decreased with increasing diffusion coefficient $D_{1, P}$. Furthermore, the CV curves for relatively large diffusion coefficient $D_{1, P}$ of $10^{-12}$ and $10^{-11} \mathrm{~m}^{2} / \mathrm{s}$ were superimposed and the capacitive current was negligibly small across the entire potential window. Indeed, Figure 4 b establishes that, for these values of $D_{1, P}$, no starvation of $\mathrm{Li}^{+}$occurred at the electrode/electrolyte interface. Instead, the concentration of intercalated lithium was uniform in the electrode and increased up to an oscillatory steady-state with a very narrow hysteresis. Then, the faradaic process was not diffusion limited, the device operated only in the faradaic regime, and the faradaic potential window was maximal, i.e., $\Delta \psi_{f}=\psi_{\max }-\psi_{\min }$.

\subsection{Effect of the Reaction Rate}

Figure 5a shows the total current density $j_{T}$ as a function of imposed cell potential $\psi_{s}(t)$ for different values of the reaction rate $k_{0}$ ranging from $10^{-7}$ to $10^{-13} \mathrm{~m}^{2.5} \mathrm{~mol}^{-0.5} \mathrm{~s}^{-1}$. Figures 
$5 \mathrm{~b}$ to $5 \mathrm{~d}$ show the capacitive $j_{C}$, faradaic $j_{F}$, and total $j_{T}$ current densities as functions of cell potential $\psi_{s}(t)$ for reaction rate $k_{0}$ of (b) $10^{-7}$, (c) $10^{-10}$, and (d) $10^{-13} \mathrm{~m}^{2.5} \mathrm{~mol}^{-0.5} \mathrm{~s}^{-1}$. In all cases, the scan rate was $v=1 \mathrm{~V} / \mathrm{s}$ and the potential window extended from $-0.8 \mathrm{~V}$ to $-0.2 \mathrm{~V}$. Figure $5 \mathrm{a}$ indicates that for reaction rates larger than $10^{-9} \mathrm{~m}^{2.5} \mathrm{~mol}^{-0.5} \mathrm{~s}^{-1}$ the CV curves were nearly identical. Figure 5b indicates that the behavior displayed involved a transition between faradaic and capacitive regimes similar to that discussed previously (Figure 2). This regime transition coincided with the charging peak in the total current density and was found to occur at the same potential for all reaction rates larger than $10^{-9}$ $\mathrm{m}^{2.5} \mathrm{~mol}^{-0.5} \mathrm{~s}^{-1}$. In other words, when the cell was not limited by the reaction rate, the latter had no effect on the $\mathrm{CV}$ curves and on the width of the faradaic potential window. Figure $5 \mathrm{c}$ shows that for a smaller reaction rate $k_{0}=10^{-10} \mathrm{~m}^{2.5} \mathrm{~mol}^{-0.5} \mathrm{~s}^{-1}$, the capacitive current dominated for the entire potential window and thus no regime transition was observed. For even lower reaction rates, Figure 5 d shows that the current was exclusively capacitive and the device behaved as an EDLC. Finally, note that Figure 5a indicates that a larger capacitance was achieved for $k_{0}=10^{-13} \mathrm{~m}^{2.5} \mathrm{~mol}^{-0.5} \mathrm{~s}^{-1}$ than for $k_{0}=10^{-10} \mathrm{~m}^{2.5} \mathrm{~mol}^{-0.5} \mathrm{~s}^{-1}$. In the latter case, the faradaic and capacitive currents had opposite signs which led to a smaller total current density.

Overall, this section established that both the pseudocapacitive electrode thickness $L_{P}$ and the diffusion coefficient $D_{1, P}$ of intercalated $\mathrm{Li}^{+}$have dramatic effects on the CV curves and on the faradaic potential window. The reaction rate constant $k_{0}$ was found to have a small effect on charge storage in the device for a reasonably large value. The interplay between the pseudocapacitve electrode parameters $L_{P}$ and $D_{1, P}$ and their respective effects on the faradaic potential window can be further studied and quantified by performing a scaling analysis. Such analysis considered a sufficiently large reaction rate $k_{0}$ so that it was not limiting. 


\section{Scaling}

\subsection{Validation of the Scaling}

In order to demonstrate the validity of the scaling described in the Analysis section, four different cases were considered with various electrode and electrolyte properties and cell dimensions. Table 2 summarizes the different values of the input parameters of Cases 1 through 4. However, these four cases featured identical dimensionless numbers governing the problem namely $v^{*}=1.13 \times 10^{-8}, \psi_{\max }^{*}=31.2, \psi_{\min }^{*}=7.79, L^{*}=3641.5, a_{2}^{*}=3.65$, $\nu_{p, 1}=1.21, \nu_{p, 2}=0.36, D_{2}^{*}=1.27, z_{2}^{*}=-1, \sigma_{P}^{*}=0.165, \sigma_{C}^{*}=0.165, L_{P}^{*}=L_{C}^{*}=36,415$, $D_{1, P}^{*}=3.8 \times 10^{-5}, c_{1, P, \max }^{*}=32.9, k_{0}^{*}=3.34 \times 10^{-8}$, and $\Delta \psi_{e q}^{*}=0$. Figures $6 \mathrm{a}$ and $6 \mathrm{~b}$ respectively show the predicted capacitive and faradaic current densities $j_{C}$ and $j_{F}$ as functions of surface potential $\psi_{s}$ for these four cases. They indicate that capacitive and faradaic current densities $j_{C}$ and $j_{F}$ varied significantly among these four cases due to the large difference in input parameters. However, Figures $6 \mathrm{c}$ and $6 \mathrm{~d}$ plot the corresponding dimensionless current densities $j_{C}^{*}$ and $j_{F}^{*}$ versus $\psi_{s}^{*}$ as defined in Equations (21) and (22), respectively. They demonstrate that the dimensionless CV curves overlapped for all four cases considered.

Similarly, Figure 6c shows the predicted $\mathrm{Li}^{+}$concentration in the pseudocapacitive electrode at the electrode/electrolyte interface, $c_{1, P}(-L, t)$ as a function of potential $\psi_{s}$ for the four cases considered. Again, the plots differed significantly both in terms of magnitude and shape. However, Figure 6 f plots the dimensionless concentration of $\mathrm{Li}^{+}$in the pseudocapacitive electrode at the electrode/electrolyte interface $c_{1, P}^{*}(-L, t)$ as a function of dimensionless potential $\psi_{s}^{*}$. It indicates that the dimensionless plots overlapped for the four cases considered. These results establish that the governing equations and boundary and initial conditions were properly scaled and that the dimensionless CV curves are uniquely determined by the 17 similarity parameters given by Equations (27).

\subsection{Scaling of Faradaic Potential Window}

The faradaic potential window $\Delta \psi_{f}$ was previously shown to be a good indicator of the performance of the hybrid pseudocapacitor simulated. Ideally, a device should operate 
exclusively in the faradaic regime to achieve large energy and power densities, as discussed previously [22]. However, most actual hybrid pseudocapacitors suffer from $\mathrm{Li}^{+}$starvation in the course of a typical cycle [6]. The fraction $R_{f}$ of the potential window dominated by faradaic reactions can be defined as

$$
R_{f}=\frac{\Delta \psi_{f}}{\Delta \psi_{s}}=\frac{\psi_{f}-\psi_{\min }}{\psi_{\max }-\psi_{\min }}
$$

According to scaling analysis, this dimensionless parameter should be a unique function of the 17 similarity parameters given by Equations (27). Here, we will limit our study to those parameters associated with the faradaic reaction and with the intercalation of $\mathrm{Li}^{+}$to obtain design guidelines for the pseudocapacitive electrode. For the sake of simplicity, the width of the potential window and the ion valencies were kept constant and equal to $\psi_{\min }=-0.8 \mathrm{~V}$, $\psi_{\max }=0.8 \mathrm{~V}$, and $z_{1}=-z_{2}=1$. Similarly, the ion diffusion coefficients in the electrolyte were those reported in Table 1 . They were large enough that the device operated in the ion diffusion-independent regime, at the scan rates considered. In other words, favorable yet realistic values of the parameters associated with the choice of the electrolyte were considered. The maximum concentration of $\mathrm{Li}^{+}$in the electrode $c_{1, P, \max }$, the equilibrium potential $\Delta \psi_{e q}$, and the reaction rate $k_{0}$ were also kept constant. First, the maximum intercalated $\mathrm{Li}^{+}$concentration $c_{1, P, \max }$ was never reached in any of our simulations. In addition, the equilibrium potential $\Delta \psi_{e q}$ was found to have a negligible effect on the behavior of the device [22]. Finally, the reaction rate constant $k_{0}$ was found to only affect the shape and magnitude of the faradaic current but not the faradaic potential window. Therefore, only the dimensionless parameters $L_{P}^{*}, D_{1, P}^{*}$, and $v^{*}$ were investigated. Their respective ranges were based on realistic values of the associated dimensional variables. The dimensionless electrode thickness $L_{P}^{*}$ ranged from 36 to $2.9 \times 10^{3}$, while the dimensionless scan rate ranged from $1.1 \times 10^{-11}$ to $1.1 \times 10^{-8}$, and the dimensionless $\mathrm{Li}^{+}$diffusion coefficient ranged from 3.8 $\times 10^{-5}$ to $1.9 \times 10^{-1}$. The latter corresponds to differences in diffusion coefficient $D_{1, P}$ between crystalline and amorphous or among eligible metal oxide materials. All the other dimensionless parameters were kept constant with the following values: $a_{2}^{*}=3.63, z_{2}^{*}=-1$, $\psi_{\text {max }}^{*}=31.2, \psi_{\text {min }}^{*}=-31.2, L^{*}=3.63 \times 10^{3}, \nu_{P 1}=3.62 \times 10^{-1}, \nu_{P 2}=1.20, D_{2}^{*}=1.27$, 
$c_{1, P, \max }^{*}=32.9, k_{0}^{*}=3.35 \times 10^{-7}$, and $\Delta \psi_{e q}^{*}=0$.

Figure 7a shows the faradaic fraction $R_{f}$ as a function of dimensionless pseudocapacitive electrode thickness $L_{P}^{*}$ for different values of dimensionless $\mathrm{Li}^{+}$diffusion coefficient $D_{1, P}^{*}$ and dimensionless scan rate $v^{*}$. For each set of $\left(D_{1, P}^{*}, v^{*}\right)$, the solid line represents a power law fitting $R_{f}$ to $L_{P}^{*}$ according to $R_{f} \propto\left(L_{p}^{*}\right)^{-1 / 2}$. This is consistent with previous results (Figure 3 ) indicating that the faradaic potential window narrowed as the electrode thickness increased.

Figure $7 \mathrm{~b}$ shows the dimensionless number $R_{f}\left(L_{P}^{*}\right)^{1 / 2}$ as a function of dimensionless $\mathrm{Li}^{+}$ diffusion coefficient $D_{1, P}^{*}$ in the pseudocapacitive electrode for different values of $L_{P}^{*}$ and $v^{*}$. Cases with identical dimensionless scan rate $v^{*}$ but different $L_{P}^{*}$ aligned along the same line according to the power law $R_{f}\left(L_{P}^{*}\right)^{1 / 2} \propto\left(D_{1, P}^{*}\right)^{1 / 4}$. This confirms that the effect of the electrode thickness was captured by the power law obtained from Figure 7a. Note that the faradaic fraction $R_{f}$ was unity for larger diffusion coefficients. In these cases, the current was exclusively faradaic and there was no $\mathrm{Li}^{+}$starvation, as reported in Section 3.3.

Figure 7c shows the dimensionless number $R_{f}\left(L_{P}^{*}\right)^{1 / 2}\left(D_{1, P}^{*}\right)^{-1 / 4}$ as a function of dimensionless scan rate $v^{*}$ for different values $D_{1, P}^{*}$ and $L_{P}^{*}$. All the cases aligned along a single line according to the power law $R_{f}\left(L_{P}^{*}\right)^{1 / 2}\left(D_{1, P}^{*}\right)^{-1 / 4} \propto\left(v^{*}\right)^{-1 / 5}$. This confirms that the effects of the electrode thickness and diffusion coefficient were correctly captured by the previous scaling.

Finally, Figure $7 \mathrm{~d}$ shows the faradaic fractionn $R_{f}$ as a function of the dimensionless parameter $\Pi_{f}$ capturing the combined effects of $L_{P}^{*}, D_{1, P}^{*}$, and $v^{*}$ and defined as

$$
\Pi_{f}=\frac{\left(D_{1, P}^{*}\right)^{1 / 4}}{\left(L_{p}^{*}\right)^{1 / 2}\left(v^{*}\right)^{1 / 5}}=\left(\frac{\tau_{d}}{\tau_{d, P}}\right)^{1 / 4}\left(\frac{\tau_{t h}}{\tau_{d}}\right)^{1 / 5}
$$

$\Pi_{f}$ can be interpreted as the product of the ratios of (i) the characteristic time for $\mathrm{Li}^{+}$ ion diffusion across the EDL, defined as $\tau_{d}=\lambda_{D}^{2} / D_{1}$, and the characteristic time for $\mathrm{Li}^{+}$ intercalation in the pseudocapacitive electrode defined as $\tau_{d, P}=L_{P}^{2} / D_{1, P}$, and of (ii) the characteristic time for reaching the thermal potential $R_{u} T / z_{1} F$ at scan rate $v$ defined as $\tau_{t h}=\left(R_{u} T / z_{1} F\right) / v$ and $\tau_{d}$. Figure $7 \mathrm{~d}$ features an intercalation-controlled regime and an 
intercalation-independent regime with a sharp transition at $\Pi_{f}=0.2$. In the intercalationcontrolled regime, the faradaic fraction $R_{f}$ was linearly proportional to $\Pi_{f}$ according to $R_{f}=2.36 \Pi_{f}$ for $\Pi_{f}<0.2$. In the intercalation-independent regime, the entire potential window was dominated by the faradaic current and $R_{f}$ was unity.

In practice, hybrid pseudocapacitors should operate in the intercalation-independent regime and the pseudocapacitive electrode should satisfy the design criterion $\Pi_{f}>0.2$. This criterion can be achieved by reducing the scan rate or charging rate so that $\mathrm{Li}^{+}$intercalation has the time to respond to changes in surface potential. This is equivalent to reducing power to maximize energy density. Alternatively, the pseudocapacitive electrode thickness can be reduced to compensate for slow $\mathrm{Li}^{+}$intercalation and to delay $\mathrm{Li}^{+}$starvation. This would correspond to reducing energy density to achieve maximum power. The most desirable solution is to select a pseudocapacitive material with fast $\mathrm{Li}^{+}$intercalation to enable the use of large electrode thickness and thus achieve both large power and energy densities. This can be obtained with crystalline structures providing fast diffusion pathways in the electrode material [43]. Actual electrodes are porous with sometimes a conductive scaffold supporting the redox active materials [5]. The above design criterion can be applied using effective properties to reflect the porosity and 3D morphology of actual electrodes. Similarly, the presence of a conductive scaffold would have to be taken into account with an effective electrical conductivity. The presence of phase transition in the electrode material may lead experimentally to additional peaks being observed in the CV curves. Materials exhibiting a phase transition upon $\mathrm{Li}^{+}$insertion are not however considered good pseudocapacitive materials [39]. In addition, nanostructuration of transition metal oxide tends to suppress the phase transition upon lithiation [44-46]. Therefore, they were excluded from the object of this study.

\section{Conclusion}

This paper presented the dimensional analysis of a continuum model simulating hybrid pseudocapacitors accounting simultaneously for (i) charge transport in the electrodes and 
electrolyte, (ii) formation of an EDL at the electrode/electrolyte interfaces, (iii) steric repulsion due to finite ion size, (iv) redox reactions at the pseudocapacitive electrode/electrolyte interface, and (v) intercalation of the reaction product in the pseudocapacitive electrode. A parametric study was conducted to identify the role of the pseudocapacitive electrode thickness, $\mathrm{Li}^{+}$diffusion coefficient, and reaction rate constant on the $\mathrm{CV}$ curves of the device for different scan rates. Two regimes were identified: a faradaic regime dominated by redox reactions and $\mathrm{Li}^{+}$intercalation and a capacitive regime dominated by EDL formation. The faradaic regime exhibited the largest current density. The transition from the faradaic to the capacitive regime observed in most cases was caused by the starvation of $\mathrm{Li}^{+}$at the electrode/electrolyte interface. The fraction of the potential window dominated by faradaic reactions was found to be a good indicator of the total capacitance of the hybrid pseudocapacitor. It increased with increasing diffusion coefficient of $\mathrm{Li}^{+}$in the pseudocapacitive electrode and with decreasing electrode thickness and scan rate. The scaling analysis led to a dimensionless parameter $\Pi_{f}$, defined in Equation (30), relating these parameters to the faradaic fraction. It also established that for $\Pi_{f}>0.2$, the entire potential window was dominated by the faradaic current and the device operated in an intercalation-independent regime. This design criterion can be used to optimize the pseudocapacitive electrode in hybrid pseudocapacitors.

\section{Acknowledgements}

This material is based upon work supported as part of the Molecularly Engineered Energy Materials, an Energy Frontier Research Center funded by the U.S. Department of Energy, Office of Science, Office of Basic Energy Sciences under Award Number DE-SC0001342.

\section{References}

[1] US Department of Energy, Basic Research Needs for Electrical Energy Storage, http: //www .osti.gov/accomplishments/documents/fullText/ACC0330.pdf, 2007.

[2] P. Simon, Y. Gogotsi, Materials for electrochemical capacitors, Nature Materials 7 (11) (2008) 845-854. 
[3] Y. Zhang, H. Feng, X. Wu, L. Wang, A. Zhang, T. Xia, H. Dong, X. Li, L. Zhang, Progress of electrochemical capacitor electrode materials: A review, International Journal of Hydrogen Energy 34 (11) (2009) 4889-4899.

[4] Z.-S. Wu, G. Zhou, L.-C. Yin, W. Ren, F. Li, H.-M. Cheng, Graphene/metal oxide composite electrode materials for energy storage, Nano Energy 1 (1) (2012) 107-131.

[5] G. Wang, L. Zhang, J. Zhang, A review of electrode materials for electrochemical supercapacitors, Chemical Society Reviews 41 (2) (2012) 797-828.

[6] V. Augustyn, J. Come, M. A. Lowe, J. W. Kim, P.-L. Taberna, S. H. Tolbert, H. D. Abruña, P. Simon, B. Dunn, High-rate electrochemical energy storage through $\mathrm{Li}^{+}$ intercalation pseudocapacitance, Nature Materials 12 (2013) 518-522.

[7] Q. Qu, P. Zhang, B. Wang, Y. Chen, S. Tian, Y. Wu, R. Holze, Electrochemical performance of $\mathrm{MnO}_{2}$ nanorods in neutral aqueous electrolytes as a cathode for asymmetric supercapacitors, Journal of Physical Chemistry C 113 (31) (2009) 14020-14027.

[8] J. W. Long, D. Bélanger, T. Brousse, W. Sugimoto, M. B. Sassin, O. Crosnier, Asymmetric electrochemical capacitors-stretching the limits of aqueous electrolytes, Material Research Society Bulletin 36 (7) (2011) 513-522.

[9] Z. Fan, J. Yan, T. Wei, L. Zhi, G. Ning, T. Li, F. Wei, Asymmetric supercapacitors based on graphene $/ \mathrm{MnO}_{2}$ and activated carbon nanofiber electrodes with high power and energy density, Advanced Functional Materials 21 (12) (2011) 2366-2375.

[10] F. Wang, S. Xiao, Y. Hou, C. Hu, L. Liu, Y. Wu, Electrode materials for aqueous asymmetric supercapacitors, RCS Advances 3 (32) (2013) 13059-13084.

[11] L. Demarconnay, E. Raymundo-Piñero, F. Béguin, Adjustment of electrodes potential window in an asymmetric carbon/ $\mathrm{MnO}_{2}$ supercapacitor, Journal of Power Sources 196 (1) (2011) 580-586. 
[12] B. Pillay, Design of Electrochemical Capacitors for Energy Storage, Ph.D. Thesis, Deptartment of Chemical Engineering, University of California, Berkeley, CA (1996).

[13] C. Lin, J. A. Ritter, B. N. Popov, R. E. White, A mathematical model of an electrochemical capacitor with double-layer and Faradaic processes, Journal of The Electrochemical Society 146 (9) (1999) 3168-3175.

[14] H. Kim, B. N. Popov, A mathematical model of oxide, carbon composite electrode for supercapacitors, Journal of the Electrochemical Society 150 (9) (2003) 1153-1160.

[15] K. Somasundaram, E. Birgersson, A. S. Mujumdar, Analysis of a model for an electrochemical capacitor, Journal of The Electrochemical Society 158 (11) (2011) 1220-1230.

[16] H. Farsi, F. Gobal, A mathematical model of nanoparticulate mixed oxide pseudocapacitors; part II: the effects of intrinsic factors, Journal of Solid State Electrochemistry 15 (1) (2011) 115-123.

[17] S. Devan, V. R. Subramanian, R. E. White, Transient analysis of a porous electrode, Journal of The Electrochemical Society 152 (5) (2005) 947-955.

[18] J. A. Staser, J. W. Weidner, Mathematical modeling of hybrid asymmetric electrochemical capacitors, Journal of The Electrochemical Society 161 (8) (2014) E3267-E3275.

[19] T. Kadyk, M. Eikerling, Charging mechanism and moving reaction fronts in a supercapacitor with pseudocapacitance, Journal of The Electrochemical Society 161 (3) (2014) A239-A246.

[20] C. Lin, B. N. Popov, H. J. Ploehn, Modeling the effects of electrode composition and pore structure on the performance of electrochemical capacitors, Journal of the Electrochemical Society 149 (2) (2002) A167-A175.

[21] G. Sikha, R. E. White, B. N. Popov, A mathematical model for a lithium-ion battery/electrochemical capacitor hybrid system, Journal of the Electrochemical Society 152 (8) (2005) A1682-A1693. 
[22] H.-L. Girard, H. Wang, A. d'Entremont, L. Pilon, Physical interpretation of cyclic voltammetry for hybrid pseudocapacitors, Journal of Physical Chemistry C 119 (21) (2015) 11349-11361.

[23] B. E. Conway, V. Birss, J. Wojtowicz, The role and utilization of pseudocapacitance for energy storage by supercapacitors, Journal of Power Sources 66 (1) (1997) 1-14.

[24] P. Guillemet, T. Brousse, O. Crosnier, Y. Dandeville, L. Athouel, Y. Scudeller, Modeling pseudo capacitance of manganese dioxide, Electrochimica Acta 67 (2012) 41-49.

[25] J. F. Rubinson, Y. P. Kayinamura, Charge transport in conducting polymers: Insights from impedance spectroscopy, Chemical Society Reviews 38 (12) (2009) 3339-3347.

[26] Y. Liu, F. Zhou, V. Ozolins, Ab Initio study of the charge-storage mechanisms in $\mathrm{RuO}_{2^{-}}$ based electrochemical ultracapacitors, Journal of Physical Chemistry C 116 (1) (2012) $1450-1457$.

[27] V. Ozolins, F. Zhou, M. Asta, Ruthenia-based electrochemical supercapacitors: Insights from first-principles calculations, Accounts of Chemical Research 46 (5) (2013) 10841093.

[28] J. Kang, S.-H. Wei, K. Zhu, Y.-H. Kim, First-principles theory of electrochemical capacitance of nanostructured materials: Dipole-assisted subsurface intercalation of lithium in pseudocapacitive $\mathrm{TiO}_{2}$ anatase nanosheets, Journal of Physical Chemistry C 115 (11) (2011) 4909-4915.

[29] D. A. Tompsett, S. C. Parker, P. G. Bruce, M. S. Islam, Nanostructuring of $\beta-\mathrm{MnO}_{2}$ : The important role of surface to bulk ion migration, Chemistry of Materials 25 (4) (2013) 536-541.

[30] H. Ding, K. G. Ray, V. Ozolins, M. Asta, Structural and vibrational properties of $\alpha$ $\mathrm{MoO}_{3}$ from van der Waals corrected density functional theory calculations, Physical Review B 85 (2012) 012104. 
[31] L. Shao, J.-W. Jeon, J. L. Lutkenhaus, Porous polyaniline nanofiber/vanadium pentoxide layer-by-layer electrodes for energy storage, Journal of Materials Chemistry A 1 (26) (2013) 7648-7656.

[32] J. Wang, J. Polleux, J. Lim, B. Dunn, Pseudocapacitive contributions to electrochemical energy storage in $\mathrm{TiO}_{2}$ (anatase) nanoparticles, Journal of Physical Chemistry C 111 (40) (2007) 14925-14931.

[33] M. C. López, G. F. Ortiz, P. Lavela, R. Alcántara, J. L. Tirado, Improved energy storage solution based on hybrid oxide materials, ACS Sustainable Chemistry \& Engineering 1 (1) (2013) 46-56.

[34] H. Wang, J. Fang, L. Pilon, Scaling laws for carbon-based electric double layer capacitors, Electrochimica Acta 109 (2013) 316-321.

[35] H. Wang, A. Thiele, L. Pilon, Simulations of cyclic voltammetry for electric double layers in asymmetric electrolytes: A generalized modified Poisson-Nernst-Planck model, Journal of Physical Chemistry C 117 (36) (2013) 18286-18297.

[36] J. H. Masliyah, S. Bhattacharjee, Electrokinetic and Colloid Transport Phenomena, John Wiley \& Sons, 2006.

[37] A. J. Bard, L. R. Faulkner, Electrochemical Methods: Fundamentals and Applications, John Wiley \& Sons, New York, NY, 2001.

[38] S. Umino, J. Newman, Diffusion of sulfuric acid in concentrated solutions, Journal of the Electrochemical Society 140 (8) (1993) 2217-2221.

[39] J. Come, V. Augustyn, J. W. Kim, P. Rozier, P.-L. Taberna, P. Gogotsi, J. W. Long, B. Dunn, P. Simon, Electrochemical kinetics of nanostructured $\mathrm{Nb}_{2} \mathrm{O}_{5}$ electrodes, Journal of The Electrochemical Society 161 (5) (2014) A718-A725.

[40] H. Wang, L. Pilon, Physical interpretation of cyclic voltammetry for measuring electric double layer capacitances, Electrochimica Acta 64 (2012) 130-139. 
[41] J. H. Masliyah, S. Bhattacharjee, Electrokinetic and Colloid Transport Phenomena, John Wiley \& Sons, Hoboken, NJ, 2006.

[42] A. A. Kornyshev, Double-layer in ionic liquids: Paradigm change?, Journal of Physical Chemistry B 111 (20) (2007) 5545-5557.

[43] J. W. Kim, V. Augustyn, B. Dunn, The effect of crystallinity on the rapid pseudocapacitive response of $\mathrm{Nb}_{2} \mathrm{O}_{5}$, Advanced Energy Materials 2 (1) (2012) 141-148.

[44] V. Augustyn, P. Simon, B. Dunn, Pseudocapacitive oxide materials for high-rate electrochemical energy storage, Energy \& Environmental Science 7 (5) (2014) 1597-1614.

[45] H.-S. Kim, J. B. Cook, S. H. Tolbert, B. Dunn, The development of pseudocapacitive properties in nanosized-MoO 2 , Journal of The Electrochemical Society 162 (5) (2015) A5083-A5090.

[46] G. A. Muller, J. B. Cook, H.-S. Kim, S. H. Tolbert, B. Dunn, High performance pseudocapacitor based on 2D layered metal chalcogenide nanocrystals, Nano letters 15 (3) (2015) 1911-1917.

[47] G. J. Janz, R. P. T. Tomkins, Nonaqueous Electrolytes Handbook, Vol. I, Academic Press, New York, NY, 1972.

[48] K. Nishikaw, Y. Fukunaka, T. Sakka, Y. H. Ogata, J. R. Selman, Measurement of $\mathrm{LiClO}_{4}$ diffusion coefficient in propylene carbonate by Moire pattern, Journal of The Electrochemical Society 153 (5) (2006) 830-834.

[49] Y.-P. Lin, N.-L. Wu, Characterization of $\mathrm{MnFe}_{2} \mathrm{O}_{4} / \mathrm{LiMn}_{2} \mathrm{O}_{4}$ aqueous asymmetric supercapacitor, Journal of Power Sources 196 (2) (2011) 851-854.

[50] E. Lim, H. Kim, C. Jo, J. Chun, K. Ku, S. Kim, H. I. Lee, I.-S. Nam, S. Yoon, K. Kang, et al., Advanced hybrid supercapacitor based on a mesoporous niobium pentoxide/carbon as high-performance anode, ACS nano 8 (9) (2014) 8968-8978. 
[51] M. Toupin, T. Brousse, D. Bélanger, Charge storage mechanism of $\mathrm{MnO}_{2}$ electrode used in aqueous electrochemical capacitor, Chemistry of Materials 16 (16) (2004) 3184-3190.

[52] D. Bélanger, T. Brousse, J. W. Long, Manganese oxides: battery materials make the leap to electrochemical capacitors, Interface 17 (1) (2008) 49-52.

[53] L. L. Zhang, R. Zhou, X. S. Zhao, Graphene-based materials as supercapacitor electrodes, Journal of Materials Chemistry 20 (29) (2010) 5983-5992.

[54] S. R. Subramanian, V. Boovaragavan, V. Ramadesigan, M. Arabandi, Mathematical model reformulation for lithium-ion battery simulations: Galvanostatic boundary conditions, Journal of The Electrochemical Society 156 (4) (2009) 260-271.

[55] A. M. Colclasure, R. J. Kee, Thermodynamically consistent modeling of elementary electrochemistry in lithium-ion batteries, Electrochimica Acta 55 (28) (2010) 8960-8973.

[56] G. M. Goldin, A. M. Colclasure, A. H. Wiedemann, R. J. Kee, Three-dimensional particle-resolved models of Li-ion batteries to assist the evaluation of empirical parameters in one-dimensional models, Electrochimica Acta 64 (2012) 118-129.

[57] J. Zhu, S. Tang, H. Xie, Y. Dai, X. Meng, Hierarchically porous $\mathrm{MnO}_{2}$ microspheres doped with homogeneously distributed $\mathrm{Fe}_{3} \mathrm{O}_{4}$ nanoparticles for supercapacitors, ACS Applied Material Interfaces 6 (20) (2014) 17637-17646.

[58] R. He, S. Chen, F. Yang, B. Wu, Dynamic diffuse double-layer model for the electrochemistry of nanometer-sized electrodes, Journal of Physical Chemistry B 110 (7) (2006) $3262-3270$. 


\section{List of Figures}

1 Schematic of one-dimensional hybrid supercapacitor consisting of a pseudocapacitive electrode and a carbon electrode with $\mathrm{LiClO}_{4}$ electrolyte in $\mathrm{PC}$. The dotted line encloses the computational domain considered. . . . . . . . . .

2 (a) Capacitive $j_{C}(t)$, faradaic $j_{F}(t)$, and total $j_{T}(t)$ current densities at the pseudocapacitive electrode and (b) concentration of intercalated $\mathrm{Li}^{+} c_{1, P}(-L, t)$ in the pseudocapacitive electrode at the electrode/electrolyte interface as functions of cell potential $\psi_{s}(t)$ at scan rate $v=1 \mathrm{~V} / \mathrm{s}$ for pseudocapacitive electrode thickness $L_{P}=50 \mathrm{~nm}$ and $\mathrm{Li}^{+}$diffusion coefficient in the electrode $D_{1, P}$ $=10^{-14} \mathrm{~m}^{2} / \mathrm{s} \ldots \ldots \ldots \ldots \ldots \ldots \ldots$ (a) Total current density $j_{T}(t)$ and (b) concentration of intercalated $\mathrm{Li}^{+}$ $c_{1, P}(-L, t)$ in the pseudocapacitive electrode at the electrode/electrolyte interface as functions of imposed cell potential $\psi_{s}(t)$ at scan rate $v=1 \mathrm{~V} / \mathrm{s}$ for pseudocapacitive electrode thickness $L_{P}$ ranging from 50 to $500 \mathrm{~nm}$ with a relatively small diffusion coefficient of $\mathrm{Li}^{+}$in the electrode $D_{1, P}=10^{-14} \mathrm{~m}^{2} / \mathrm{s}$. 35 (a) Total current density $j_{T}(t)$ and (c) concentration of intercalated $\mathrm{Li}^{+} c_{1, P}(-L, t)$ in the pseudocapacitive electrode of thickness $L_{P}=500 \mathrm{~nm}$ at the electrode/electrolyte interface as functions of imposed cell potential $\psi_{s}(t)$ at scan rate $v=1 \mathrm{~V} / \mathrm{s}$ for diffusion coefficient $D_{1, P}$ ranging from $10^{-14}$ to $10^{-11} \mathrm{~m}^{2} / \mathrm{s}$. 36 (a) Total current density $j_{T}$ as a function of cell potential $\psi_{s}(t)$ at the pseudocapacitive electrode for five different values of the reaction rate $k_{0}$. Capacitive $j_{C}$, faradaic $j_{F}$, and total $j_{T}$ current densities as functions of cell potential $\psi_{s}(t)$ at the pseudocapacitive electrode for reaction rate constant $k_{0}$ equals to (b) $10^{-7}$, (c) $10^{-10}$, and (d) $10^{-13} \mathrm{~m}^{2.5} \mathrm{~mol}^{-0.5} \mathrm{~s}^{-1}$. . . . . . . . . . . . .

6 Predicted dimensional currents (a) $j_{C}$, (b) $j_{F}$, and (c) concentration $c_{1, P}$ as functions of imposed potential $\psi_{s}$ along with the corresponding dimensionless currents (d) $j_{C}^{*}$, (e) $j_{F}^{*}$, and (f) concentration $c_{1, P}^{*}$ as functions of dimensionless imposed potential $\psi_{s}^{*}$ for cases 1 through 4 summarized in Table 2. . . . . . (a) Faradaic fraction $R_{f}$ as a function of dimensionless electrode thickness $L_{P}^{*}$ for different values of the dimensionless diffusion coefficient of $\mathrm{Li}^{+}$inside the pseudocapacitive electrode $D_{1, P}^{*}$. (b) $R_{f}\left(L_{P}^{*}\right)^{1 / 2}$ as a function of the dimensionless $\mathrm{Li}^{+}$diffusion coefficient $D_{1, P}^{*}$. (c) $R_{f}\left(L_{P}^{*}\right)^{1 / 2}\left(D_{1, P}^{*}\right)^{-1 / 4}$ as a function of dimensionless scan rate $v^{*}$. (d) Faradaic fraction $R_{f}$ as a function of dimensionless parameter $\Pi_{f}$ defined in Equation (30) . . . . . . . . . . . 
Figure 1: Schematic of one-dimensional hybrid supercapacitor consisting of a pseudocapacitive electrode and a carbon electrode with $\mathrm{LiClO}_{4}$ electrolyte in PC. The dotted line encloses the computational domain considered.

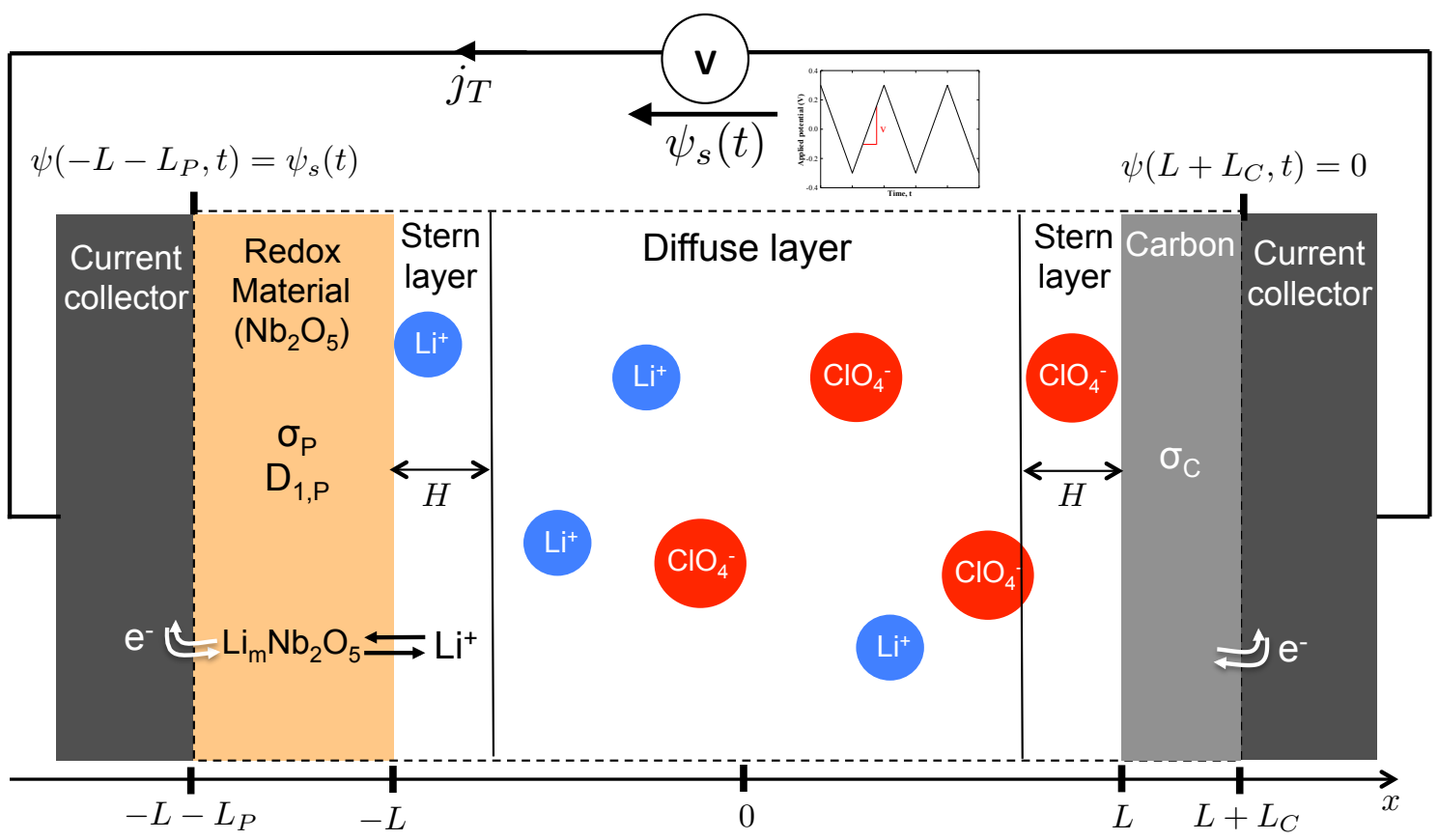


(a)

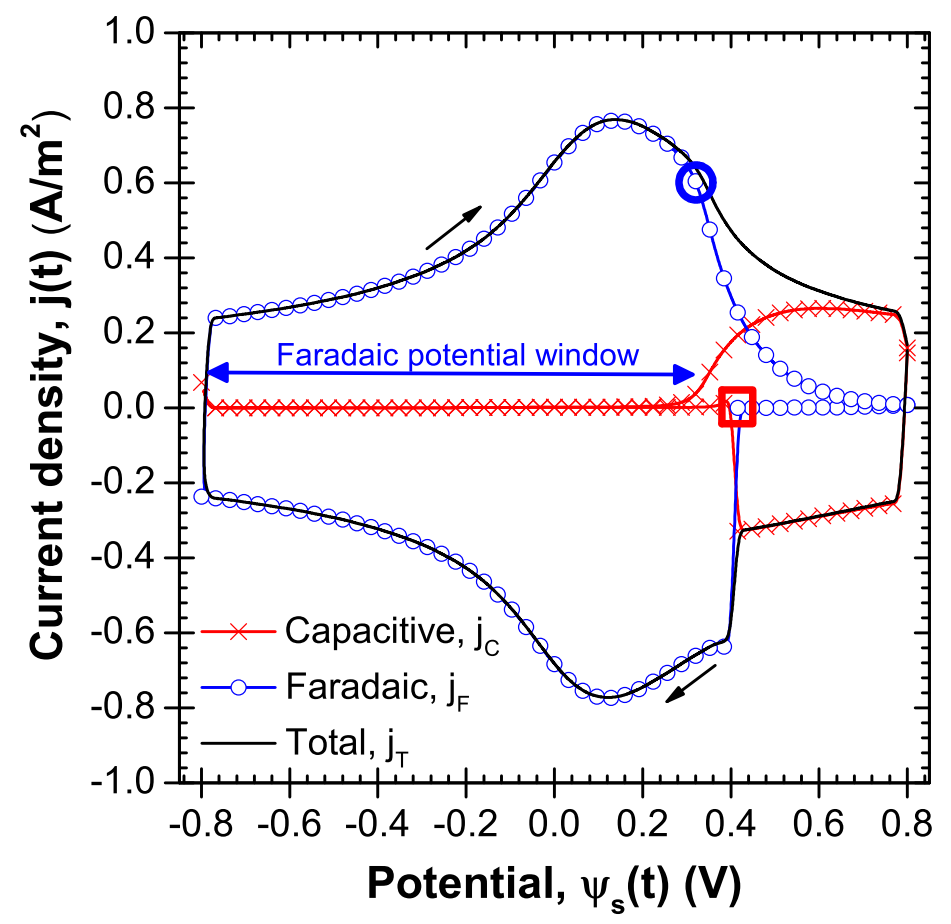

(b)

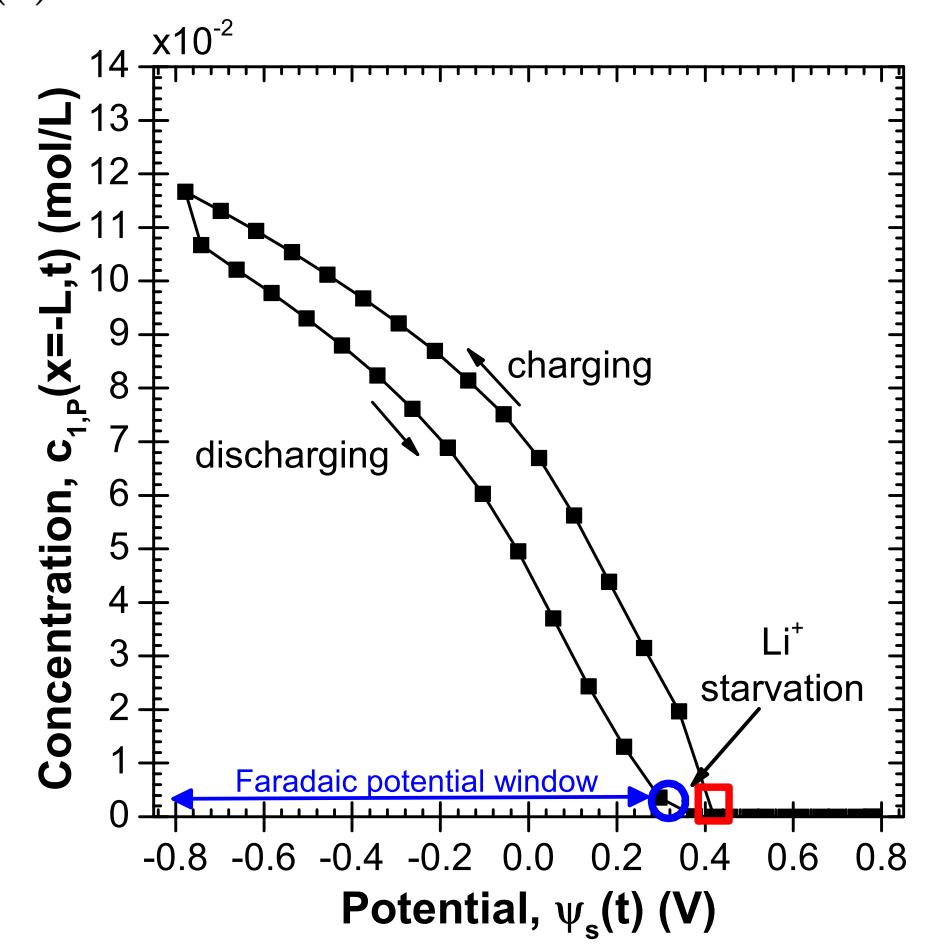

Figure 2: (a) Capacitive $j_{C}(t)$, faradaic $j_{F}(t)$, and total $j_{T}(t)$ current densities at the pseudocapacitive electrode and (b) concentration of intercalated $\mathrm{Li}^{+} c_{1, P}(-L, t)$ in the pseudocapacitive electrode at the electrode/electrolyte interface as functions of cell potential $\psi_{s}(t)$ at scan rate $v=1 \mathrm{~V} / \mathrm{s}$ for pseudocapacitive electrode thickness $L_{P}=50 \mathrm{~nm}$ and $\mathrm{Li}^{+}$diffusion coefficient in the electrode $D_{1, P}=10^{-14} \mathrm{~m}^{2} / \mathrm{s}$. 
(a)

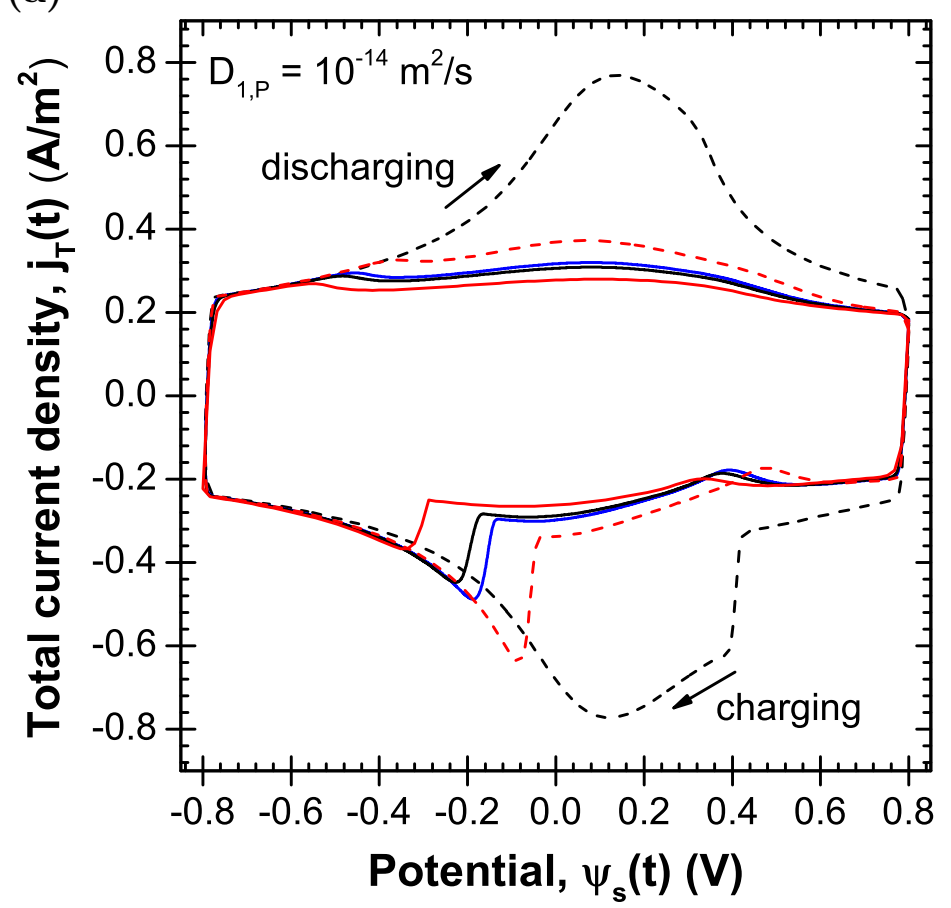

(b)

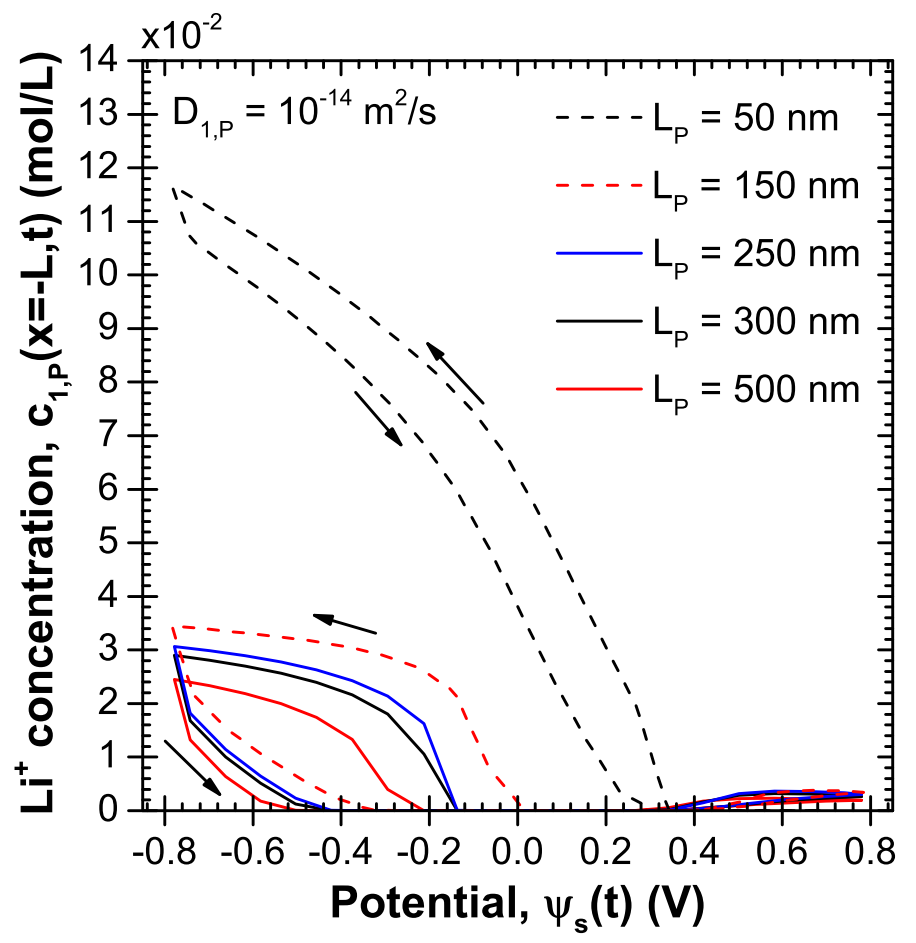

Figure 3: (a) Total current density $j_{T}(t)$ and (b) concentration of intercalated $\mathrm{Li}^{+} c_{1, P}(-L, t)$ in the pseudocapacitive electrode at the electrode/electrolyte interface as functions of imposed cell potential $\psi_{s}(t)$ at scan rate $v=1 \mathrm{~V} / \mathrm{s}$ for pseudocapacitive electrode thickness $L_{P}$ ranging from 50 to $500 \mathrm{~nm}$ with a relatively small diffusion coefficient of $\mathrm{Li}^{+}$in the electrode $D_{1, P}=10^{-14} \mathrm{~m}^{2} / \mathrm{s}$. 
(a)

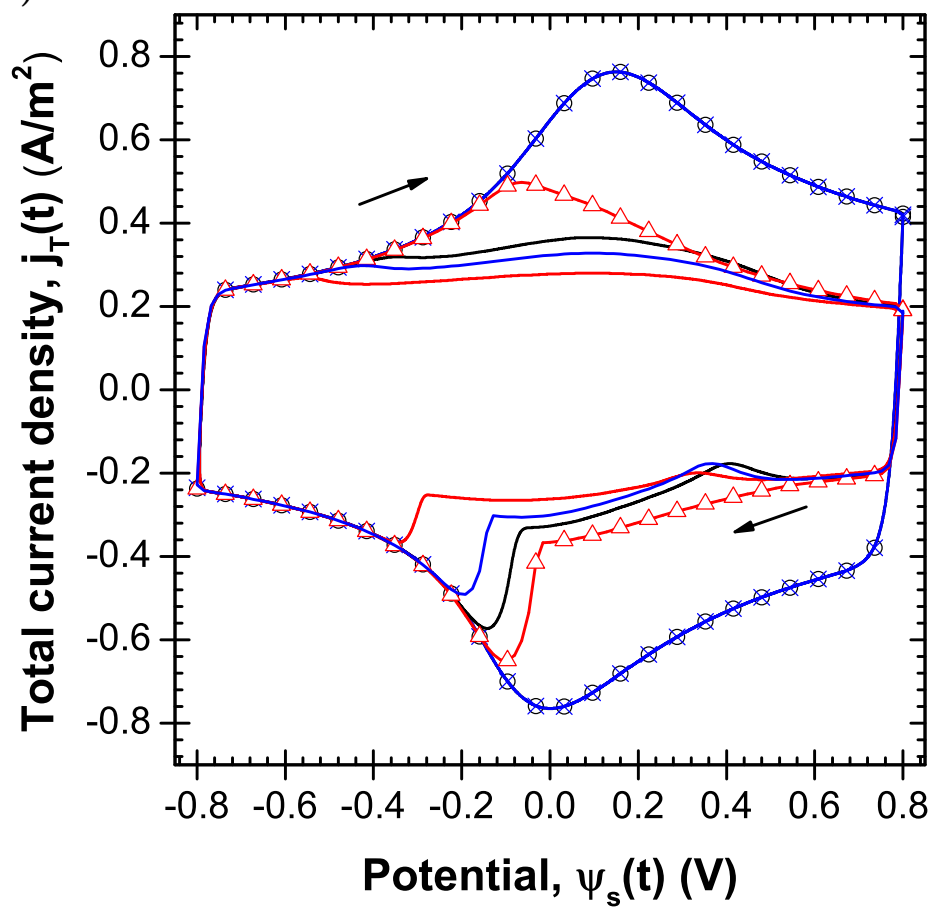

(b)

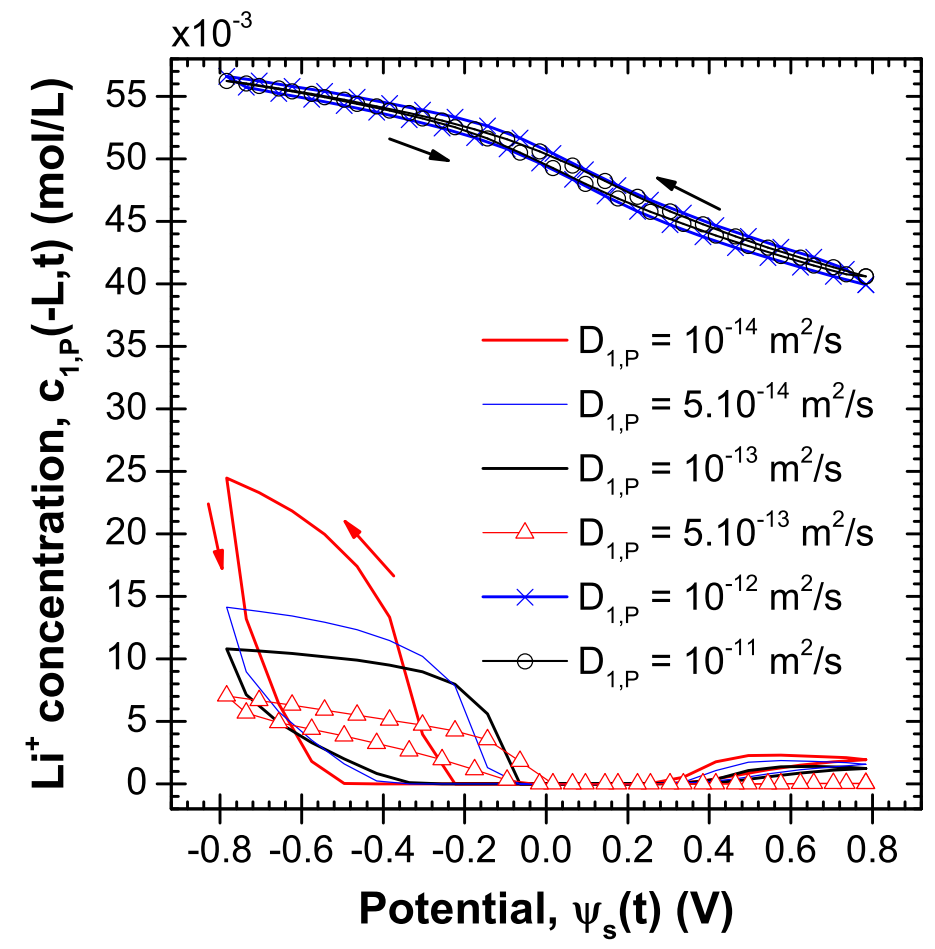

Figure 4: (a) Total current density $j_{T}(t)$ and (c) concentration of intercalated $\mathrm{Li}^{+} c_{1, P}(-L, t)$ in the pseudocapacitive electrode of thickness $L_{P}=500 \mathrm{~nm}$ at the electrode/electrolyte interface as functions of imposed cell potential $\psi_{s}(t)$ at scan rate $v=1 \mathrm{~V} / \mathrm{s}$ for diffusion coefficient $D_{1, P}$ ranging from $10^{-14}$ to $10^{-11} \mathrm{~m}^{2} / \mathrm{s}$. 
(a)

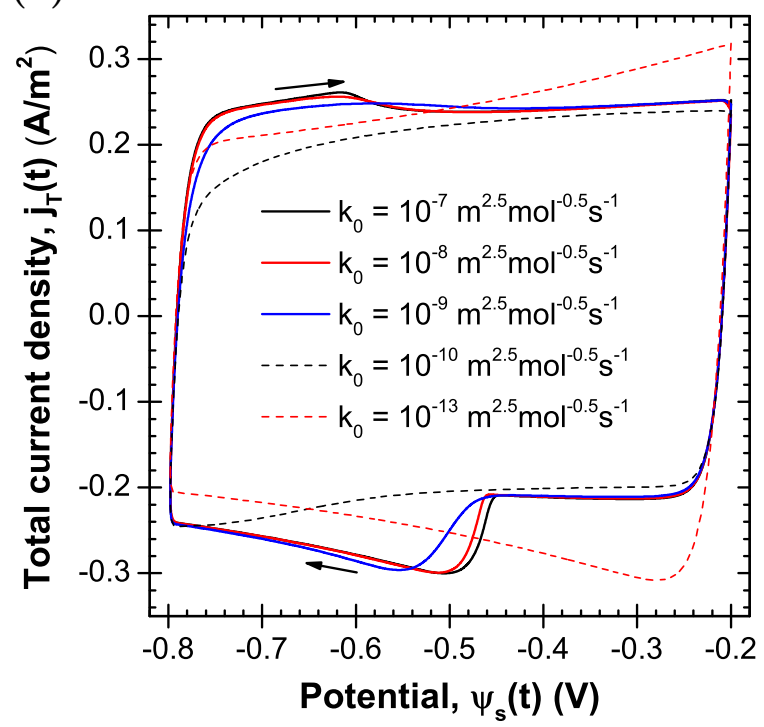

(c)

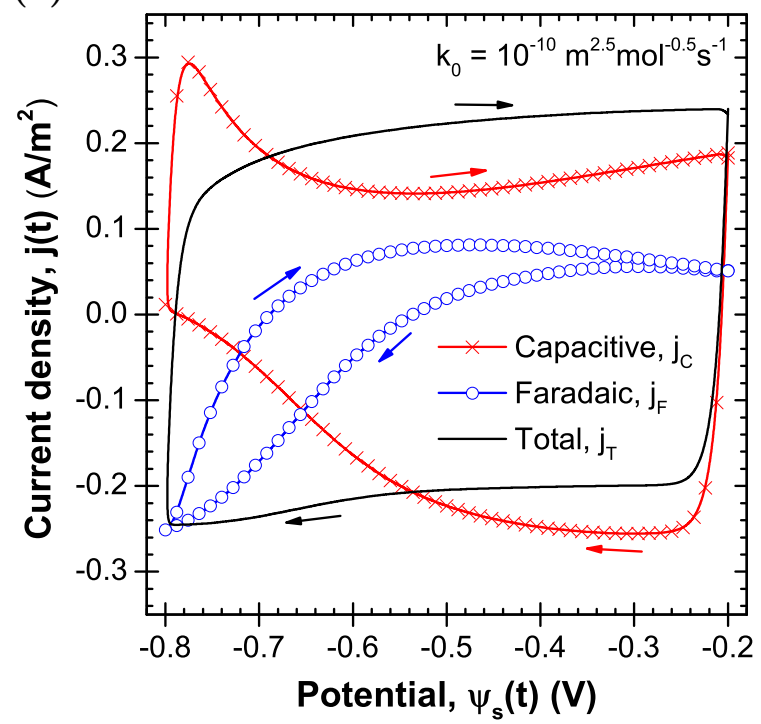

(b)

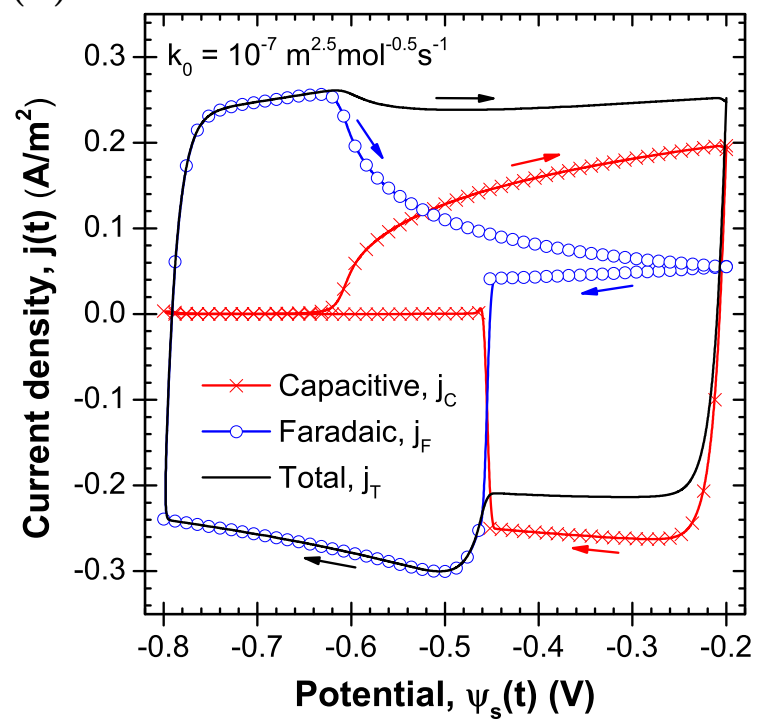

(d)

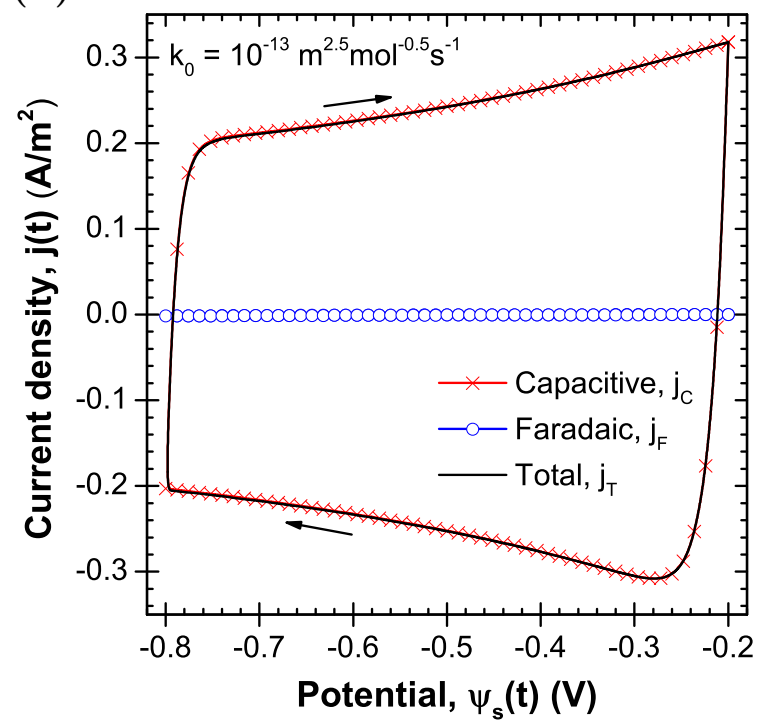

Figure 5: (a) Total current density $j_{T}$ as a function of cell potential $\psi_{s}(t)$ at the pseudocapacitive electrode for five different values of the reaction rate $k_{0}$. Capacitive $j_{C}$, faradaic $j_{F}$, and total $j_{T}$ current densities as functions of cell potential $\psi_{s}(t)$ at the pseudocapacitive electrode for reaction rate constant $k_{0}$ equals to (b) $10^{-7}$, (c) $10^{-10}$, and (d) $10^{-13} \mathrm{~m}^{2.5} \mathrm{~mol}^{-0.5} \mathrm{~s}^{-1}$. 
(a) Dimensional

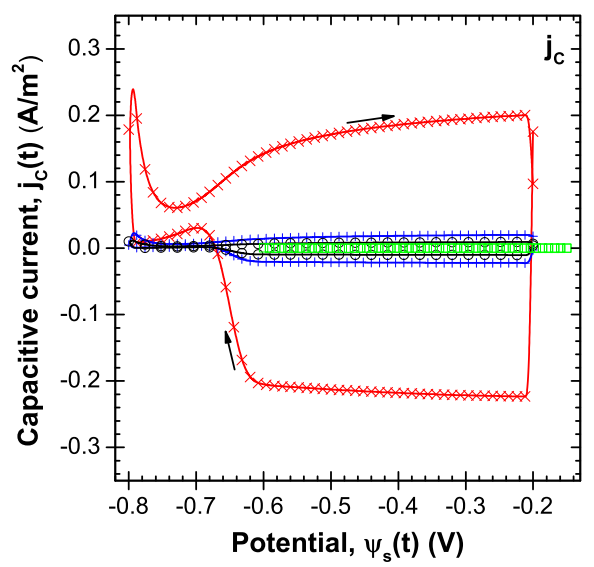

(b)

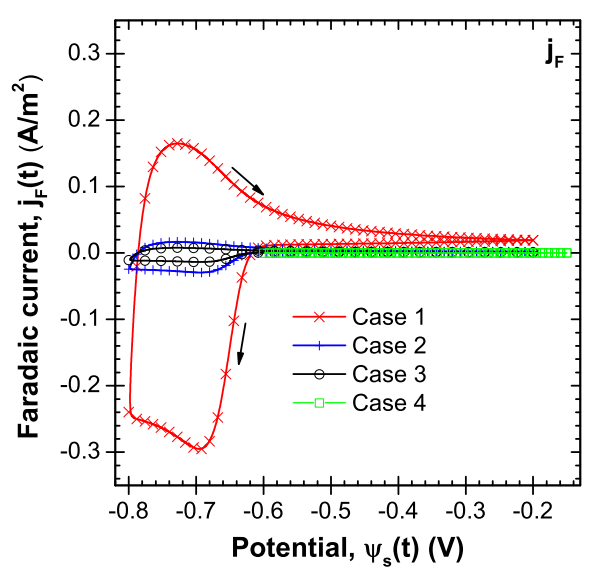

(c)

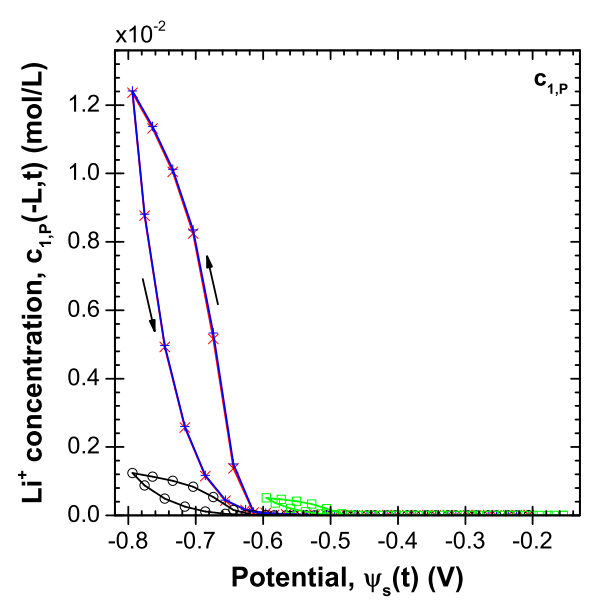

(d) Dimensionless

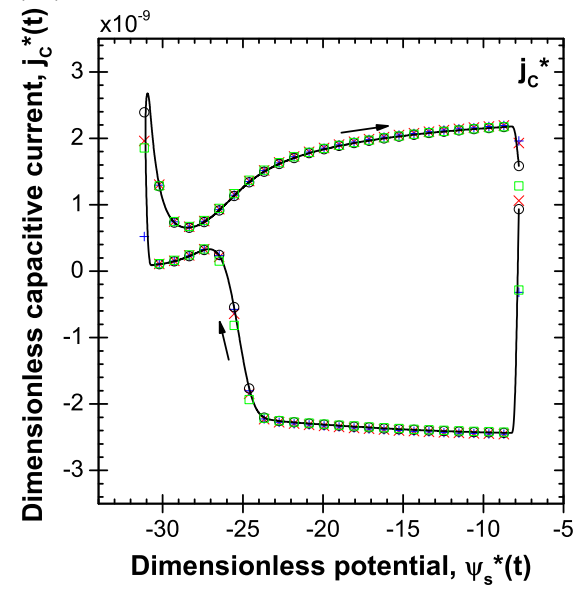

(e)

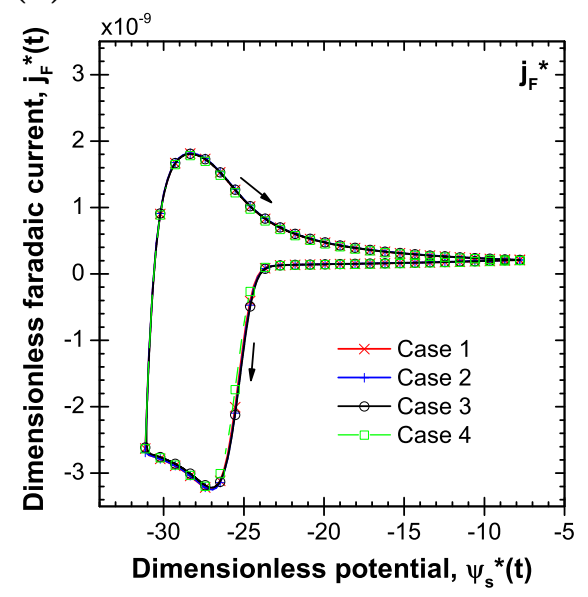

(f)

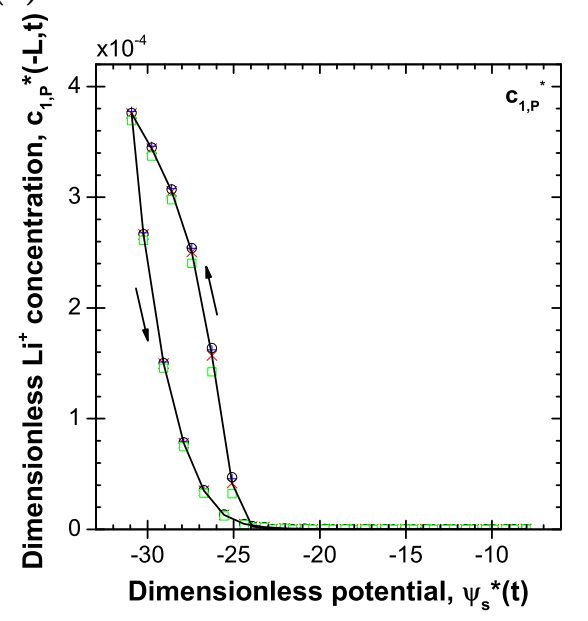

Figure 6: Predicted dimensional currents (a) $j_{C}$, (b) $j_{F}$, and (c) concentration $c_{1, P}$ as functions of imposed potential $\psi_{s}$ along with the corresponding dimensionless currents (d) $j_{C}^{*}$, (e) $j_{F}^{*}$, and (f) concentration $c_{1, P}^{*}$ as functions of dimensionless imposed potential $\psi_{s}^{*}$ for cases 1 through 4 summarized in Table 2. 
(a)

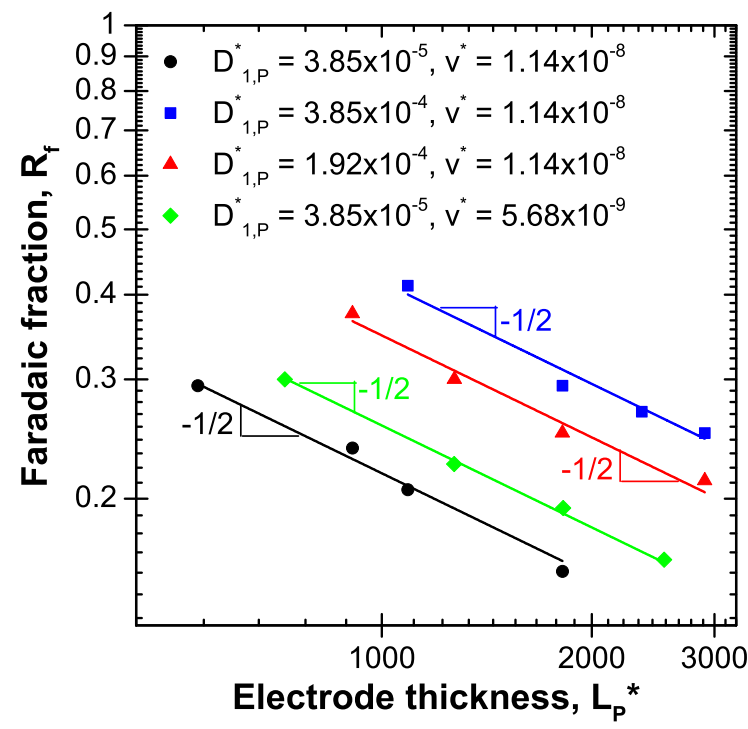

(c)

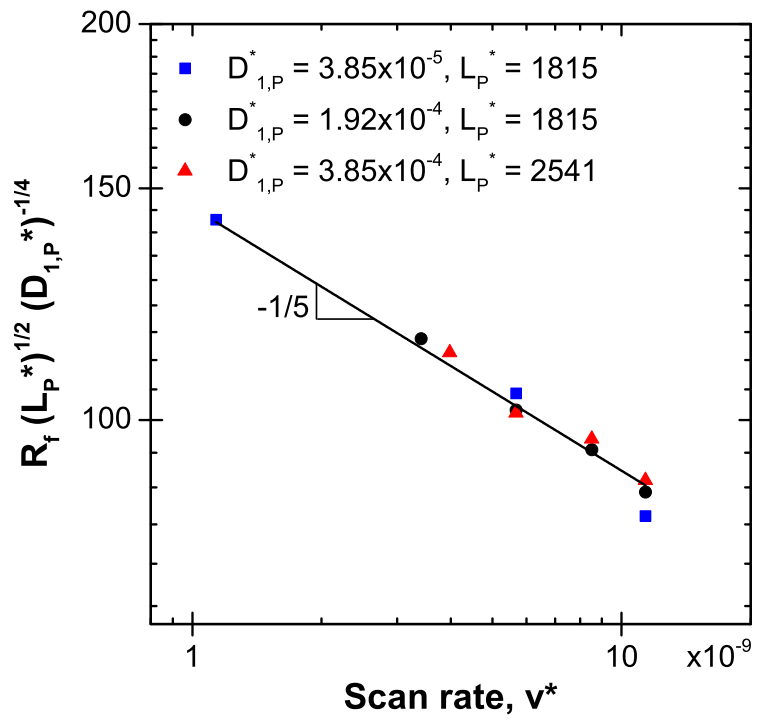

(b)

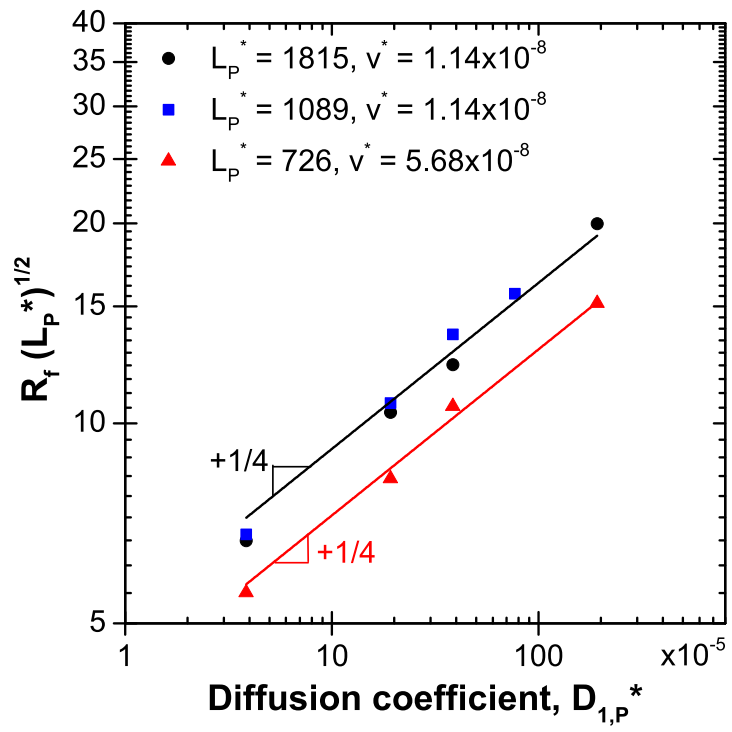

(d)

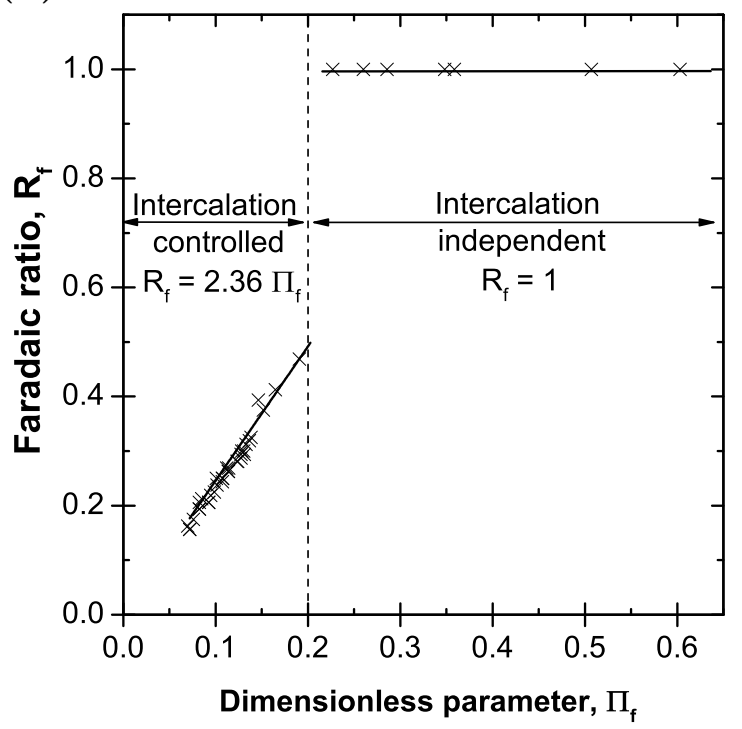

Figure 7: (a) Faradaic fraction $R_{f}$ as a function of dimensionless electrode thickness $L_{P}^{*}$ for different values of the dimensionless diffusion coefficient of $\mathrm{Li}^{+}$inside the pseudocapacitive electrode $D_{1, P}^{*}$. (b) $R_{f}\left(L_{P}^{*}\right)^{1 / 2}$ as a function of the dimensionless $\mathrm{Li}^{+}$diffusion coefficient $D_{1, P}^{*}$. (c) $R_{f}\left(L_{P}^{*}\right)^{1 / 2}\left(D_{1, P}^{*}\right)^{-1 / 4}$ as a function of dimensionless scan rate $v^{*}$. (d) Faradaic fraction $R_{f}$ as a function of dimensionless parameter $\Pi_{f}$ defined in Equation (30). 


\section{List of Tables}

1 Electrode and electrolyte properties and dimensions used in the simulations reported in this study. . . . . . . . . . . . . . . . . . . 41

2 Parameters used in CV simulations reported in Figure 6 with $\Delta \psi_{e q}=0 \mathrm{~V}$ and $z_{1}=-z_{2}=1$ for all cases considered. In these four cases, the dimensionless numbers identified in Equation (27) were identical and equal to $v^{*}=1.13 \times$ $10^{-8}, \psi_{\max }^{*}=31.2, \psi_{\min }^{*}=7.79, L^{*}=3641.5, a_{2}^{*}=3.65, \nu_{p, 1}=1.21, \nu_{p, 2}=$ $0.36, D_{2}^{*}=1.27, z_{2}^{*}=-1, \sigma_{P}^{*}=0.16, \sigma_{C}^{*}=12, L_{P}^{*}=L_{C}^{*}=36415, D_{1, P}^{*}=$ $3.8 \times 10^{-5}, k_{0}^{*}=3.34 \times 10^{-8}$, and $\Delta \psi_{e q}^{*}=0 \ldots \ldots \ldots \ldots$ 
Table 1: Electrode and electrolyte properties and dimensions used in the simulations reported in this study.

\begin{tabular}{|c|c|c|c|c|}
\hline Parameters & Symbol & Value & Unit & Ref. \\
\hline Temperature & $\mathrm{T}$ & 298 & $\mathrm{~K}$ & \\
\hline Relative permitttivity & $\epsilon_{r}$ & 64.4 & & [47] \\
\hline $\mathrm{Li}^{+}$ion diameter & $a_{1}$ & 0.67 & $\mathrm{~nm}$ & {$[48]$} \\
\hline $\mathrm{ClO}_{4}^{-}$ion diameter & $a_{2}$ & 1.0 & $\mathrm{~nm}$ & [48] \\
\hline $\mathrm{Li}^{+}$valency & $z_{1}$ & 1 & & \\
\hline $\mathrm{ClO}_{4}^{-}$valency & $z_{2}$ & -1 & & \\
\hline $\mathrm{Li}^{+}$diffusion coefficient & $D_{1}$ & $2.6 \times 10^{-10}$ & $\mathrm{~m}^{2} / \mathrm{s}$ & [48] \\
\hline $\mathrm{ClO}_{4}^{-}$diffusion coefficient & $D_{2}$ & $3.3 \times 10^{-10}$ & $\mathrm{~m}^{2} / \mathrm{s}$ & {$[48]$} \\
\hline Stern layer thickness & $\mathrm{H}$ & 0.5 & $\mathrm{~nm}$ & {$[35]$} \\
\hline Initial bulk ion concentration & $c_{1, \infty}=c_{2, \infty}$ & 1 & $\mathrm{~mol} / \mathrm{L}$ & {$[33,49,50]$} \\
\hline Initial intercalated $\mathrm{Li}^{+}$concentration & $c_{1, P, 0}$ & 1 to 5 & $\mathrm{~mol} / \mathrm{L}$ & {$[51]$} \\
\hline Pseudocapacitive electrode conductivity & $\sigma_{P}$ & $10^{-5}$ & $\mathrm{~S} / \mathrm{m}$ & [52] \\
\hline Carbon electrode conductivity & $\sigma_{C}$ & 5 & $\mathrm{~S} / \mathrm{m}$ & [53] \\
\hline Reaction rate & $k_{0}$ & $10^{-7}$ to $10^{-13}$ & $\mathrm{~m}^{2.5} \mathrm{~mol}^{-0.5} \mathrm{~s}^{-1}$ & {$[54-56]$} \\
\hline Intercalated $\mathrm{Li}^{+}$diffusion coefficient & $D_{1, P}$ & $10^{-14}$ to $10^{-10}$ & $\mathrm{~m}^{2} / \mathrm{s}$ & {$[55]$} \\
\hline Half electrolyte thickness & $L$ & 1 & $\mu \mathrm{m}$ & \\
\hline Pseudocapacitive electrode thickness & $L_{P}$ & 20 to 500 & $\mathrm{~nm}$ & {$[32,57]$} \\
\hline Carbon electrode thickness & $L_{C}$ & 20 to 500 & $\mathrm{~nm}$ & \\
\hline Equilibrium potential difference & $\Delta \psi_{e q}$ & 0 & $\mathrm{~V}$ & {$[58]$} \\
\hline Transfer coefficient & $\alpha$ & $1 / 2$ & & \\
\hline Scan rate & $v$ & $10^{-3}$ to 10 & $\mathrm{~V} / \mathrm{s}$ & {$[33,49,50]$} \\
\hline Potential window & $\psi_{\max }-\psi_{\min }$ & 1.6 & $\mathrm{~V}$ & {$[33,49,50]$} \\
\hline
\end{tabular}


Table 2: Parameters used in CV simulations reported in Figure 6 with $\Delta \psi_{e q}=0 \mathrm{~V}$ and $z_{1}=-z_{2}=1$ for all cases considered. In these four cases, the dimensionless numbers identified in Equation (27) were identical and equal to $v^{*}=1.13 \times 10^{-8}, \psi_{\max }^{*}=31.2, \psi_{\min }^{*}=7.79, L^{*}=3641.5, a_{2}^{*}=3.65, \nu_{p, 1}=1.21, \nu_{p, 2}=0.36$, $D_{2}^{*}=1.27, z_{2}^{*}=-1, \sigma_{P}^{*}=0.16, \sigma_{C}^{*}=12, L_{P}^{*}=L_{C}^{*}=36415, D_{1, P}^{*}=3.8 \times 10^{-5}, k_{0}^{*}=3.34 \times 10^{-8}$, and $\Delta \psi_{e q}^{*}=0$.

\begin{tabular}{lcccc}
\hline Parameters & Case 1 & Case 2 & Case 3 & Case 4 \\
\hline $\boldsymbol{v}(\mathbf{V} / \mathbf{s})$ & 1 & 0.1 & 0.215 & 0.0091 \\
$\boldsymbol{\psi}_{\boldsymbol{m a x}}(\mathbf{V})$ & 0.8 & 0.8 & 0.8 & 0.6 \\
$\boldsymbol{\psi}_{\boldsymbol{m i n}}(\mathbf{V})$ & 0.2 & 0.2 & 0.2 & 0.15 \\
$\mathbf{T}(\mathbf{K})$ & 298 & 298 & 298 & 223.5 \\
$\boldsymbol{a}_{\mathbf{1}}(\mathbf{n m})$ & 0.67 & 0.67 & 1.44 & 1.92 \\
$\boldsymbol{a}_{\mathbf{2}}(\mathbf{n m})$ & 1.0 & 1.0 & 2.16 & 2.88 \\
$\boldsymbol{D}_{\mathbf{1}}\left(\mathbf{m}^{2} / \mathbf{s}\right)$ & $2.6 \times 10^{-10}$ & $2.6 \times 10^{-11}$ & $2.6 \times 10^{-10}$ & $2.6 \times 10^{-11}$ \\
$\boldsymbol{D}_{\mathbf{2}}\left(\mathbf{m}^{2} / \mathbf{s}\right)$ & $3.3 \times 10^{-10}$ & $3.3 \times 10^{-11}$ & $3.3 \times 10^{-10}$ & $3.3 \times 10^{-11}$ \\
$\boldsymbol{c}_{\mathbf{1}, \boldsymbol{\infty}}(\mathbf{m o l} / \mathbf{L})$ & 1.0 & 1.0 & 0.1 & 0.042 \\
$\boldsymbol{\epsilon}_{\boldsymbol{r}}$ & 78.5 & 78.5 & 36.4 & 36.4 \\
$\boldsymbol{L}(\mu \mathbf{m})$ & 1.0 & 1.0 & 2.15 & 2.87 \\
$\boldsymbol{\sigma}_{\boldsymbol{P}}(\mathbf{S} / \mathbf{m})$ & 0.069 & 0.0069 & 0.0069 & $3.88 \times 10^{-4}$ \\
$\boldsymbol{\sigma}_{\boldsymbol{C}}(\mathbf{S} / \mathbf{m})$ & 5 & 5 & 5 & 5 \\
$\boldsymbol{L}_{\boldsymbol{P}}=\boldsymbol{L}_{\boldsymbol{C}}(\mu \mathbf{m})$ & 10 & 10 & 21.5 & 28.7 \\
$\boldsymbol{D}_{\mathbf{1}, \boldsymbol{P}}\left(\mathbf{m}^{2} / \mathbf{s}\right)$ & $10^{-14}$ & $10^{-15}$ & $10^{-14}$ & $10^{-15}$ \\
$\boldsymbol{c}_{\mathbf{1}, \boldsymbol{P}, \boldsymbol{m a x}}(\mathbf{m o l} / \mathbf{L})$ & 32.9 & 32.9 & 3.29 & 1.39 \\
$\boldsymbol{k}_{\mathbf{0}}\left(\mathbf{m}^{2.5} \mathbf{m o l} \mathbf{m o . 5}^{-0} \mathbf{s}^{-1}\right)$ & $10^{-9}$ & $10^{-10}$ & $1.47 \times 10^{-9}$ & $1.69 \times 10^{-10}$ \\
\hline
\end{tabular}

